\title{
Kv4.3 Channel Dysfunction Contributes to Trigeminal Neuropathic Pain Manifested with Orofacial Cold Hypersensitivity in Rats
}

\author{
Hirosato Kanda, Jennifer Ling, Ya-Ting Chang, Ferhat Erol, Viacheslav Viatchenko-Karpinski, Akihiro Yamada, \\ 단 Koichi Noguchi, and Jianguo G. Gu \\ Department of Anesthesiology and Perioperative Medicine, University of Alabama at Birmingham, Birmingham, Alabama 35294
}

Trigeminal neuropathic pain is the most debilitating pain disorder but current treatments including opiates are not effective. A common symptom of trigeminal neuropathic pain is cold allodynia/hyperalgesia or cold hypersensitivity in orofacial area, a region where exposure to cooling temperatures are inevitable in daily life. Mechanisms underlying trigeminal neuropathic pain manifested with cold hypersensitivity are not fully understood. In this study, we investigated trigeminal neuropathic pain in male rats following infraorbital nerve chronic constrictive injury (ION-CCI). Assessed by the orofacial operant behavioral test, ION-CCI animals displayed orofacial cold hypersensitivity. The cold hypersensitivity was associated with the hyperexcitability of small-sized trigeminal ganglion (TG) neurons that innervated orofacial regions. Furthermore, ION-CCI resulted in a reduction of A-type voltage-gated $\mathrm{K}^{+}$currents (IA currents) in these TG neurons. We further showed that these smallsized TG neurons expressed Kv4.3 voltage-gated $\mathrm{K}^{+}$channels, and Kv4.3 expression in these cells was significantly downregulated following ION-CCI. Pharmacological inhibition of Kv4.3 channels with phrixotoxin-2 inhibited IA-currents in these TG neurons and induced orofacial cold hypersensitivity. On the other hand, pharmacological potentiation of Kv4.3 channels amplified IA currents in these TG neurons and alleviated orofacial cold hypersensitivity in ION-CCI rats. Collectively, Kv4.3 downregulation in nociceptive trigeminal afferent fibers may contribute to peripheral cold hypersensitivity following trigeminal nerve injury, and Kv4.3 activators may be clinically useful to alleviate trigeminal neuropathic pain.

Key words: cold hyperalgesia; Kv4.3 channels; nerve injury; orofacial pain; trigeminal neuropathic pain; voltage-gated $\mathrm{K}^{+}$ channels

Significance Statement

Trigeminal neuropathic pain, the most debilitating pain disorder, is often triggered and exacerbated by cooling temperatures. Here, we created infraorbital nerve chronic constrictive injury (ION-CCI) in rats, an animal model of trigeminal neuropathic pain to show that dysfunction of Kv4.3 voltage-gated $\mathrm{K}^{+}$channels in nociceptive-like trigeminal ganglion (TG) neurons underlies the trigeminal neuropathic pain manifested with cold hypersensitivity in orofacial regions. Furthermore, we demonstrate that pharmacological potentiation of Kv4.3 channels can alleviate orofacial cold hypersensitivity in ION-CCI rats. Our results may have clinical implications in trigeminal neuropathic pain in human patients, and Kv4.3 channels may be an effective therapeutic target for this devastating pain disorder.

\section{Introduction}

Trigeminal neuropathic pain such as trigeminal neuralgia (Bendtsen et al., 2019), traumatic trigeminal neuropathy (Devine et al., 2018), and postherpetic neuralgia (Feller et al., 2017) are devastating pain

\footnotetext{
Received July 22, 2020; revised Jan. 8, 2021; accepted Jan. 11, 2021.

Author contributions: K.N. and J.G.G. designed research; H.K., J.L., Y.-T.C., F.E., and V.V.-K. performed research; H.K., J.L., Y.-T.C., F.E., V.V.-K., A.Y., and J.G.G. analyzed data; J.G.G. wrote the paper.

This work was supported by National Institutes of Health Grants DE018661 and DE023090 (to J.G.G.).

The authors declare no competing financial interests.

Correspondence should be addressed to Jianguo G. Gu at jianguogu@.uabmc.edu.

https://doi.org/10.1523/JNEUROSCI.2036-20.2021

Copyright $\odot 2021$ the authors
}

conditions caused by lesions or diseases of trigeminal nerves. The neuropathic pain occurs in orofacial regions when the V2 branches of trigeminal ganglion nerves (V2 TG) are involved. Trigeminal neuropathic pain is clinically treated with medications such as anticonvulsants, surgery interventions, and alternative medicine (Christoforou, 2018; Devine et al., 2018), but these treatments were not effective for many of the patients (Christoforou, 2018). Mechanisms underlying trigeminal neuropathic pain are not well understood. This lack of knowledge has prevented us from effectively treating trigeminal neuropathic pain.

A common symptom of trigeminal neuropathic pain is orofacial cold allodynia/hyperalgesia or cold hypersensitivity (Zakrzewska 
and McMillan, 2011; Zakrzewska, 2013). In orofacial regions, stimuli such as cooling temperatures are mainly conveyed by thin afferent fibers of trigeminal V2 branches. TRPM8 channels have been identified as principal cold transducers expressed in a sub-population of small-sized afferent fibers (McKemy et al., 2002; Peier et al., 2002; McKemy, 2005; Bautista et al., 2007; Takashima et al., 2007). TRPM8 channels are shown to be involved in cold allodynia/hyperalgesia following spinal nerve injury (Colburn et al., 2007). Enhanced expression of TRPM8 in nociceptive afferent neurons have been suggested to be a mechanism underlying cold allodynia/ hyperalgesia (Xing et al., 2007; Stucky et al., 2009; Piña et al., 2019). Consistently, genetic deletion of TRPM8-expressing afferent nerves not only abolished aversive responses to noxious cold, but also significantly attenuated cold pain associated with inflammation and peripheral nerve injury (Knowlton et al., 2013). Although blockers of TRPM8 channels could alleviate allodynia/hyperalgesia in animal neuropathic pain models (Zuo et al., 2013), systemic effects by TRPM8 blockers on body's thermal regulation is a concern for their clinical uses.

Sensory stimuli including skin cooling are encoded by action potentials (APs) at afferent nerve endings following initial transduction. Therefore, membrane excitability of afferent nerves significantly affects peripheral sensitivity to cold stimuli. Membrane excitability of nociceptive afferent nerves are controlled by multiple types of ion channels including voltage-gated $\mathrm{K}^{+}$channels (Waxman and Zamponi, 2014) and two pore-domain $\mathrm{K}^{+}$channels (K2P; Gada and Plant, 2019). Previous studies have found that, in addition to TRPM8 channels, cold sensitivity in somatosensory neurons is also controlled by Kv1 type of voltage-gated $\mathrm{K}^{+}$channels (Madrid et al., 2009; González et al., 2017) and TASK-3 as well as TRESK types of K2P channels (MorenillaPalao et al., 2014; Castellanos et al., 2020; Sharif-Naeini, 2020). Dysregulated ion channel expression in response to nerve injury and inflammation can result in enhanced neuronal excitability that underlies inflammatory and neuropathic pain (Waxman and Zamponi, 2014). A-type $\mathrm{K}^{+}$currents (IA) have drawn particular attention for their essential role in controlling neuronal membrane excitability (Zemel et al., 2018). IA currents are mediated by $\mathrm{Kv1.4}, \mathrm{Kv3} 3, \mathrm{Kv3} .4, \mathrm{Kv} 4.1, \mathrm{Kv} 4.2$, and $\mathrm{Kv} 4.3$ voltagegated $\mathrm{K}^{+}$channels, and dorsal root ganglion (DRG) neurons mainly express Kv1.4, Kv3.4, Kv4.1, and Kv4.3 (Zemel et al., 2018). IA currents become reduced following chronic compression of DRGs, which may contribute to neuronal hyperexcitability and hyperalgesia (Tan et al., 2006). A recent study shows that IA currents in joint sensory neurons are reduced and excitability of these neurons enhanced in a murine model of arthritis (Qu and Caterina, 2016). Kv4.3 channels are found to be mainly expressed in nociceptive DRG neurons (Huang et al., 2005; Phuket and Covarrubias, 2009). Nociceptive DRG neurons also express Kv3.4 and Kv1.4 channels (Rasband et al., 2001; Huang et al., 2005; Phuket and Covarrubias, 2009). In a neuropathic pain model induced by spinal nerve ligation in rats, expression of both Kv3.4 and Kv4.3 in DRG neurons are found to be reduced (Chien et al., 2007). In a rat model of hand-arm vibration syndrome, both Kv1.4 and Kv4.3 expression are downregulated in nociceptive DRG neurons (Conner et al., 2016; Alvarez et al., 2019). The potential roles of these IA channels in trigeminal neuropathic pain have not been well studied. Trigeminal neuropathic pain in human patients are often caused by trigeminal nerve injury following chronic compressions of trigeminal nerves by arteries, tumors, and other pathologic factors (Feller et al., 2017; Christoforou, 2018; Devine et al., 2018; Bendtsen et al., 2019). Therefore, in this study, we used infraorbital nerve chronic constrictive injury (ION-CCI) rat model to study roles of IA cannels in trigeminal neuropathic pain.

\section{Materials and Methods \\ ION-CCI model}

Male Sprague Dawley rats (350-500 g) were used. Animal care and use conformed to NIH guidelines for care and use of experimental animals. Experimental protocols were approved by the Institutional Animal Care and Use Committee (IACUC) of the University of Alabama at Birmingham. Animals first underwent a two-week presurgical adaptation training for orofacial operant test (see below, Orofacial operant behavioral assessments). The rats were then divided into two groups, the sham and the ION-CCI group. In the ION-CCI group a chronic constriction nerve injury model was created by ligation of the trigeminal V2 branches (infraorbital nerves) as described in our previously studies (Zuo et al., 2013; Abd-Elsayed et al., 2015). The infraorbital nerve ligation was performed bilaterally unless otherwise indicated. In brief, the rats were anesthetized with intraperitoneal injection of ketamine/xylazine cocktail $(100 \mathrm{mg} / \mathrm{kg})$. The hairs above both eyes were shaved and the rat head was immobilized. For each side, a 2 -cm curvilinear incision was made superior to the orbital cavity. A meticulous dissection was made, and the muscle and fascia were retracted laterally. The infraorbital nerve could be found $\sim 1 \mathrm{~cm}$ down against the floor of the maxillary bone. The nerve was freed from the surrounding connective tissues and two ligatures were made $\sim 5 \mathrm{~mm}$ apart with a 5-0 absorbable chromic gut suture Superion. The incision was closed with 6-0 non-absorbable braided silk suture. For the sham group, rats underwent similar surgical procedures but without any ligatures.

\section{Orofacial operant behavioral assessments}

Rats were placed in a standard rat cage with a plastic divider to create two rooms, the testing room and the companion room. In the anterior aspect of the cage there was an Ugo Basile apparatus with a drinking window for the rat head to enter and acquire a reward (milk) located on the opposing aspect of the drinking window. Nestle Carnation Sweetened Condensed Milk was diluted with deionized water to $30 \%$ and placed in a cylindrical plastic container with metal nipple drinker. The apparatus also consisted of a thermal module in the drinking window. An infrared photo-beam was built on the exterior aspect of the drinking window and wired to a computer to automatically detect head accessing the feeding tube. Animals initially underwent two weeks of adaptation training sessions before surgery. The adaptation training was preceded by a $12-\mathrm{h}$ fasting period. The training was started by placing a rat in the testing room and another one in the companion room. After the rats were given $10 \mathrm{~min}$ to familiarize themselves with their environment, the drinking window was opened and the testing rat was subsequently timed for $10 \mathrm{~min}$ to allow drinking the milk. After two weeks of the presurgical adaptation training, the rats were divided into the sham and the ION groups, and sham and ION-CCI surgeries were performed as described in the previous section. After a two-week healing period, the rats underwent a two-week period of postsurgical adaption training performed in the same manner as the presurgical adaptation training.

Subsequently, experiments were performed with the thermal module during postoperative period of four to eight weeks. The thermal module consisted a metal tubing that surrounded the drinking window and the tubing contained circulating ethylene glycol (Sigma-Aldrich) made in a 50/50 mixture with distilled water. The temperatures of the circulating ethylene glycol solution were controlled by a thermal circulating bath unit. The distance between the metal tube and the nipple of the milk bottle was $14 \mathrm{~mm}$. For cooling stimulation, thermal module was set at $5^{\circ} \mathrm{C}$ in the present study. The day before each orofacial operant test, the animal's orofacial region was shaved. Similar to the adaptation training, all experiments were preceded by a 12 -h fasting period, $10 \mathrm{~min}$ for the rats to be familiarized with testing environment, and a subsequent $10 \mathrm{~min}$ to allow for orofacial operant behavioral assessment. During orofacial operant test, the cheeks of animals were subjected to the cold stimuli by contacting the metal tube as it poked its head through the opening of the thermal module to obtain the milk. To test the effects of the Kv4.3 
activator NS5806 (Tocris) on cold sensitivity of ION-CCI rats, animals were administered with NS5806 (1.67 mg/kg, i.p) $30 \mathrm{~min}$ before the orofacial operant tests. At different days, these animals were also administered with vehicle (10\% DMSO in saline) as control and then orofacial operant tests were performed in the same manner. In a different set of experiments, effects of the Kv4.3 blocker phrixotoxin-2 (Alomone Labs) on orofacial cold sensitivity was examined in rats that did not undergo ION-CCI. In these experiments, one group of animals were injected intracutaneously in orofacial regions with phrixotoxin-2 $(2 \mu \mathrm{M}, 50 \mu \mathrm{l}$, phrixotoxin-injected group) and another group of animals were injected intracutaneously in orofacial regions with vehicle solution (50 $\mu \mathrm{l}$ PBS, control group). The injection of the aforementioned testing drugs in the orofacial region was conducted in isoflurane-anesthetized animals. Orofacial operant tests were then preformed $60 \mathrm{~min}$ following the administration of phrixotoxin-2. For each operant test mentioned above, the events of head pokes were detected by the infrared photobeam, recorded by a computer, and analyzed by the Oro software (Ugo Basile). This computer software recorded and analyzed variables of the rat's behavior including the total time the beam was broken also defined as the total contact time, and the count, which also was described as total numbers of contacts.

Patch-clamp recordings from orofacial cutaneous TG afferent neurons in ex vivo whole-mount TGs

TG neurons innervating the skin of orofacial regions (orofacial cutaneous TG afferent neurons) were retrogradely labeled by intracutaneous injections of 1,1'-dioctadecy-3,3,3',3'-tetramethylindocarbocyanine perchlorate (DiI; Molecular Probe) into the skin of orofacial regions. In brief, rats were anesthetized with isoflurane and $5 \mu \mathrm{l}$ DiI solution $(25 \mathrm{mg} / \mathrm{ml})$ was injected into the skin of ipsilateral cheeks of ION-CCI or sham rats using a microsyringe. One week after the DiI injection, rats were deeply anesthetized with isoflurane and decapitated. Ipsilateral TGs with their attached nerve bundles $(\sim 15 \mathrm{~mm})$ were rapidly dissected out and placed in the ice cold Leibovitz-15 medium (Corning cellgro). Connective tissues on the surface of the TG were carefully removed with a fine forceps. TGs with their nerve bundles were then affixed in a recording chamber by a tissue anchor and submerged in a normal Krebs' solution that contained the following: $117 \mathrm{~mm} \mathrm{NaCl}, 3.5 \mathrm{~mm} \mathrm{KCl}, 2.5$ $\mathrm{mm} \mathrm{CaCl}_{2}, 1.2 \mathrm{~mm} \mathrm{MgCl}_{2}, 1.2 \mathrm{~mm} \mathrm{NaH} \mathrm{PO}_{4}, 25 \mathrm{~mm} \mathrm{NaHCO}$, and 11 $\mathrm{mm}$ glucose. The Krebs' solution was saturated with $95 \% \mathrm{O}_{2}$ and $5 \%$ $\mathrm{CO}_{2}$, had pH 7.35 and osmolarity of $324 \mathrm{mOsm}$, and at room temperature of $24^{\circ} \mathrm{C}$. TGs were exposed to $0.05 \%$ dispase II and $0.05 \%$ collagenase in the Krebs' solution for $5 \mathrm{~min}$, and then TG was continuously perfused at $2 \mathrm{ml} / \mathrm{min}$ with Krebs' solution. DiI-labeled neurons were first identified under epifluorescence illumination. Then under the DIC-IR microscope, whole-cell patch-clamp recordings were performed on the small-sized DiI-labeled V2 TG neurons which had diameters $<35 \mu \mathrm{m}$. The electrode resistance after filling recording electrode internal solution ranged from 5 to $6 \mathrm{M} \Omega$. Recording electrode internal solution contained the following: $105 \mathrm{~mm} \mathrm{~K}$-gluconate, $35 \mathrm{~mm} \mathrm{KCl}, 0.5 \mathrm{~mm} \mathrm{CaCl}_{2}, 2.4 \mathrm{~mm}$ $\mathrm{MgCl}_{2}, 5$ mм EGTA, $10 \mathrm{~mm}$ HEPES, $5 \mathrm{~mm} \mathrm{Na} \mathrm{N}_{2} \mathrm{ATP}$, and $0.33 \mathrm{~mm}$ GTPTRIS salt; the $\mathrm{pH}$ of the solution was adjusted to 7.35 with $\mathrm{KOH}$.

The junction potential of the recording condition was $+12 \mathrm{mV}$ as calculated based on ionic concentrations of internal and bath solutions by using the pCLAMP 10 software (Molecular Devices). After establishing whole-cell access, recordings were performed under the voltageclamp configuration with neurons held at $-72 \mathrm{mV}$ (voltage command of $-60 \mathrm{mV}$ ). Voltage steps were applied from -102 to $+58 \mathrm{mV}$ (voltage command of -90 to $+70 \mathrm{mV}$ ) with increments of $10 \mathrm{mV}$ each step and a step duration of $500 \mathrm{~ms}$. Unless otherwise indicated, membrane voltages mentioned in the texts for this set of experiments have been corrected for the calculated junction potentials of $+12 \mathrm{mV}$.

To determine membrane excitability of the small-sized DiI-labeled V2 TG neurons, under the current-clamp configuration step currents were applied to the neurons from -100 to $1800 \mathrm{pA}$ with increments of $20 \mathrm{pA}$ per step and the duration of each pulse was $1 \mathrm{~s}$. The excitability of the small-sized V2 TG neurons was also tested by applying ramp currents from 0 to $1500 \mathrm{pA}$ over 1-s under the current-clamp configuration. Neuronal excitability was characterized by AP threshold, rheobase, and numbers of APs evoked by a two-time rheobase stepwise current or by a ramp current. Other passive and active electrophysiological parameters were analyzed including cell capacitance, input resistance, resting membrane potential, AP amplitude and AP width. Input resistance was measured with a $10-\mathrm{mV}$ voltage step from the membrane holding voltage of $-72 \mathrm{mV}$. AP amplitude or AP upstroke was measured from AP threshold to AP peak. AP width was measured as the duration from $50 \%$ of AP upstroke to $50 \%$ of AP repolarization.

To classify the types of small-sized DiI-labeled V2 TG neurons based on the conduction velocity of their afferent fibers, APs were evoked at the peripheral end of trigeminal V2 branches (the infraorbital nerves) using a suction stimulation electrode. The suction stimulation electrode was fire-polished and had its tip size of $\sim 1 \mathrm{~mm}$ in diameter. The peripheral end of the infraorbital nerve was aspirated into the suction stimulation electrode by a negative pressure. APs were evoked by monophasic square wave pulses generated by an electronic stimulator (Master-8) and delivered via a stimulation isolator and the suction stimulation electrode to the peripheral ends of the infraorbital nerves. The duration of the stimulation pulse was $50 \mu$ s. Conduction velocity was calculated based on the latency of APs and the length of axons. The latency of APs was measured from the time of stimulation that was marked by a stimulation artifact to the time when AP was initiated at the recorded TG neuron. The length of the axon was the distance between the stimulation site and the recording site. Signals of voltage-clamp experiments were amplified using an Axopach 200B amplifier, filtered at $2 \mathrm{kHz}$ and sampled at $10 \mathrm{kHz}$ using pCLAMP 10 software (Molecular Devices). Signals of current-clamp recordings were low-pass filtered at $2 \mathrm{kHz}$ and sampled at $50 \mathrm{kHz}$.

Patch-clamp recordings of IA currents from small-sized V2 TG neurons V2 TG neurons from TGs of sham and ION-CCI groups were acutely dissociated and used for patch-clamp recordings to determine IA currents isolated from total outward currents. In brief, rats were deeply anesthetized with isoflurane and killed by decapitation. V2 parts of TGs were dissected out and incubated with dispase II $(5 \mathrm{mg} / \mathrm{ml})$ and Type I collagenase $(2 \mathrm{mg} / \mathrm{ml})$ in $2-\mathrm{ml}$ bath solution at $35^{\circ} \mathrm{C}$ for $45 \mathrm{~min}$. The bath solution was the same one used for cell perfusion in electrophysiology experiments (see below). After a rinse, isolated V2 TGs were triturated to dissociate the neurons in the bath solution, and the dissociated V2 TG neurons were plated on glass coverslips coated with poly-D-lysine and maintained at room temperature. Whole-cell patch-clamp recordings were performed within 1-4 h after cell plating. For patch-clamp recordings, the V2 TG neurons were perfused with normal bath solution flowing at $1 \mathrm{ml} / \mathrm{min}$ in a $0.5 \mathrm{ml}$ chamber on the stage of an Olympus IX70 microscope. The bath solution contained the following: $150 \mathrm{~mm} \mathrm{NaCl}, 5$ $\mathrm{mm} \mathrm{KCl}, 2 \mathrm{~mm} \mathrm{MgCl}_{2}, 2 \mathrm{~mm} \mathrm{CaCl}$, $10 \mathrm{~mm}$ glucose, and $10 \mathrm{~mm}$ HEPES, $\mathrm{pH} 7.4$, osmolarity of $320 \mathrm{mOsm}$. The recording electrode internal solution contained the following: $135 \mathrm{~mm} \mathrm{~K}$-gluconate, $0.5 \mathrm{~mm} \mathrm{CaCl}, 2 \mathrm{~mm}$ $\mathrm{MgCl}_{2}, 5 \mathrm{~mm} \mathrm{KCl}, 5 \mathrm{~mm}$ EGTA, $10 \mathrm{~mm}$ HEPES, $2 \mathrm{~mm} \mathrm{Na}_{2}$-ATP, and 0.5 mM Na $\mathrm{Na}_{2}$-GTP, pH 7.2 and osmolarity of 315-325 mOsm. Recording electrode resistance was $\sim 5 \mathrm{M} \Omega$ after filling the recording electrode internal solution. The series resistance of each recording was below $30 \mathrm{M} \Omega$ and was not compensated. Recording signals were amplified with Axopatch 200B (Molecular Devices), filtered at $2 \mathrm{kHz}$, and sampled at $5 \mathrm{kHz}$ using pCLAMP 10 (Molecular Devices). All recordings were made from the small-sized V2 TG neurons (diameter $<35 \mu \mathrm{m}$ ) at the room temperature of $24^{\circ} \mathrm{C}$. IA currents were determined using a method described in our previous study (Sarria et al., 2012). In brief, for $\mathrm{K}^{+}$current isolation, bath solution contained the following: $130 \mathrm{~mm}$ choline- $\mathrm{Cl}$, $5 \mathrm{~mm} \mathrm{KCl}, 1 \mathrm{~mm} \mathrm{MgCl}$, $10 \mathrm{~mm}$ glucose, and $10 \mathrm{~mm}$ HEPES 10, pH 7.3 and $330 \mathrm{mOsm} . \mathrm{K}^{+}$currents were activated by 480 -ms depolarizing voltage steps (command voltages) to potentials ranging between -35 and $+45 \mathrm{mV}$ in $10 \mathrm{mV}$ increments preceded by a 1-s prepulse voltage step to either $-100 \mathrm{mV}$ (for total current) or $-10 \mathrm{mV}$ [non-inactivating (IK) current only]. IA currents were then isolated by subtraction of the noninactivating outward currents from the total outward currents. To test effects of Kv4.3 activator NS5806 on IA currents, IA currents were determined before and $10 \mathrm{~min}$ following the application of $50 \mu \mathrm{M}$ NS5806. In a subset of experiments, IA currents were recorded from small-sized V2 
A

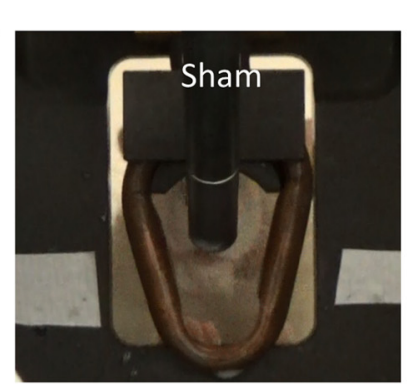

D Sham

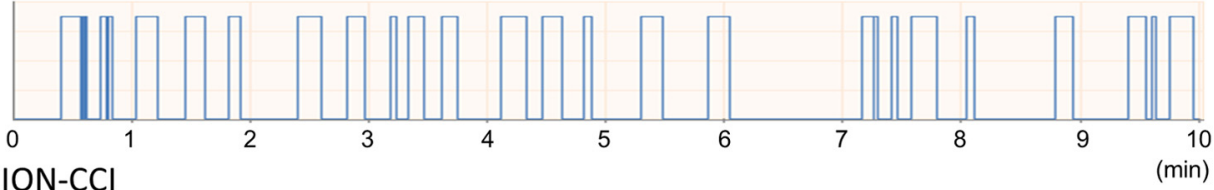

E $\mathrm{ION}-\mathrm{CCl}$

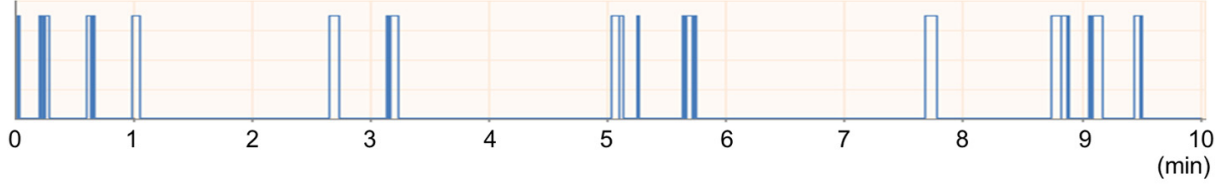

$\mathbf{F}$

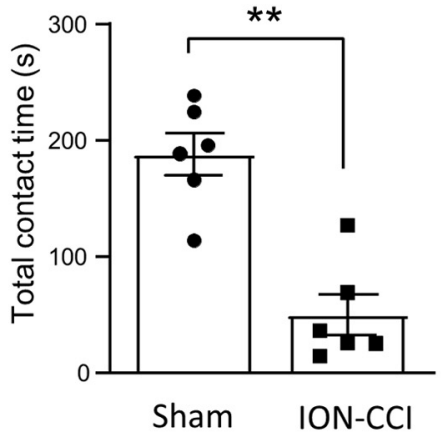

G
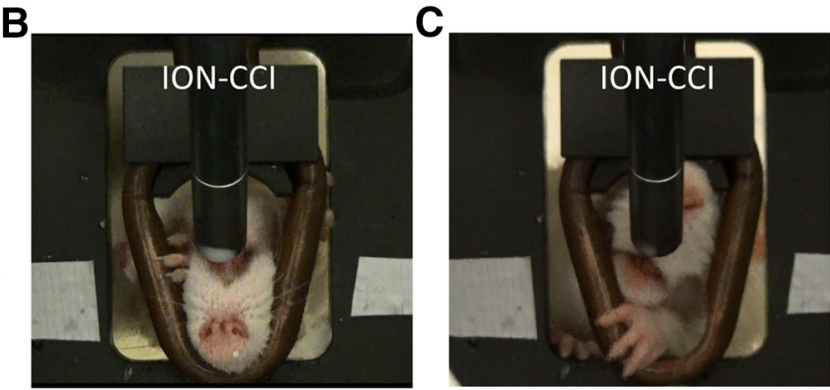

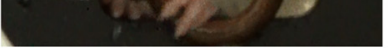

H
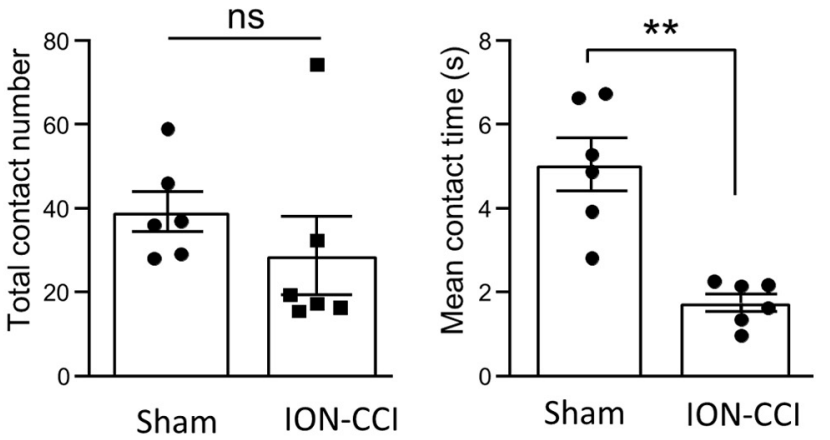

Figure 1. Orofacial cold hypersensitivity following ION-CCI determined by the orofacial operant assessment. $A$, Image shows a drinking posture of a sham rat. $B$, Image shows a drinking posture with a reversed head position in an ION-CCI rat. C, Image shows another unusual drinking posture in a different ION-CCI rat. The rat used its front paw to guard its orofacial region while drinking. In $A-C$, the thermal module consisting of a metal pipe in the drinking window was set at $5^{\circ} C$. $\boldsymbol{D}, \boldsymbol{E}$, Two sample traces show the automatic recordings of drinking behavior in a 10 -min testing session in a sham rat $(\boldsymbol{D})$ and a ION-CCI rat $(\boldsymbol{E})$. The thermal module temperature was set at $5^{\circ} \mathrm{C}$. $\boldsymbol{F}-\boldsymbol{H}$, Total contact time $(\boldsymbol{F})$, contact numbers $(\boldsymbol{G})$, and mean contact time $(\boldsymbol{H})$ of the orofacial operant test determined with thermal module set at $5^{\circ} \mathrm{C}$ in the sham group ( $n=6$, open bars) and the ION-CCI group $\left(n=6\right.$, closed bar). Data represent mean $\pm \mathrm{SEM}$; NS, no significant difference, ${ }^{* *} p<0.01$.

TG neurons in ex vivo whole-mount TGs rather than from the acutely dissociated TG neurons, and the recording conditions were the same as those performed in acutely dissociated V2 TG neurons. In this subset of experiments, effects of Kv4.3 blocker phrixotoxin-2 on IA currents were examined by determining IA currents before and $10 \mathrm{~min}$ following the application of $70 \mathrm{~nm}$ phrixotoxin-2. During the aforementioned patchclamp recordings, we continually monitored whole-cell current transients in response to membrane seal tests with $10 \mathrm{mV}$ testing pulses. The recording data were discarded if the whole-cell current transients were significantly altered during the course of recordings. In addition, before the test of effects of phrixotoxin-2 on IA currents, we performed control experiments and found that IA currents were stable and no run down occurred over the recording period of at least $45 \mathrm{~min}$. Signals of voltageclamp experiments were amplified using an Axopach 200B or Multiclamp 700B amplifier, filtered at $2 \mathrm{kHz}$ and sampled at $10 \mathrm{kHz}$ using pCLAMP 10 software (Molecular Devices). Unless otherwise indicated as commend voltages, membrane voltages mentioned in the texts in this set of experiments have been corrected for the calculated junction potentials of $28.6 \mathrm{mV}$.

\section{Immunohistochemistry}

TGs were harvested from animals five to six weeks following bilateral ligation of infraorbital nerves or sham surgery and used for immunostaining to determine Kv4.3 channel expression. In brief, animals were anesthetized with ketamine/xylazine (100:10 mg/kg, i.p), transcardially exsanguinated with $0.1 \mathrm{M}$ PBS, and perfused with $4 \%$ paraformaldehyde (PFA) in $0.1 \mathrm{M}$ PBS. Ipsilateral TGs were removed and placed in $30 \%$ sucrose for cryoprotection for two nights. The TGs were then embedded in OCT compound (Baxter Scientific) and cut on a cryostat (Leica Biosystems) into $10-\mu \mathrm{m}$ sections. The sections of TGs were thawmounted onto slides and allowed for air-dry. For each slide, three TG sections from sham animals (control) and three TG sections from IONCCI animals were mounted on the same slide. These TG sections were then encircled together with hydrophobic resin (PAP Pen, The Binding Site) so that the two groups were paired for immunostaining. The slidemounted sections were rinsed with the BupHTM Modified Dulbecco's PBS (ThermoFisher Scientific) three times, and then sequentially incubated at room temperature with ethanol solutions at the concentrations of $50 \%$ for $10 \mathrm{~min}, 70 \%$ for $10 \mathrm{~min}$, and $50 \%$ for $10 \mathrm{~min}$. The slides were rinsed three times with PBS and sections incubated with $10 \%$ normal goat serum in PBS for $1 \mathrm{~h}$ at room temperature. The sections were incubated with a polyclonal rabbit anti-Kv4.3 antibody (1:2000 diluted with $5 \%$ normal goat serum in PBS; Abcam) at $4^{\circ} \mathrm{C}$ for 2 nights. Following three rinses with PBS solution, the sections were incubated with a secondary antibody for 1 night at room temperature. The secondary antibody (1:1000 in 5\% normal goat serum in PBS) was a goat anti-rabbit IgG conjugated with Alexa Fluor 594 (ThermoFisher Scientific). The sections were rinsed three times with PBS solution, cover-slipped with 


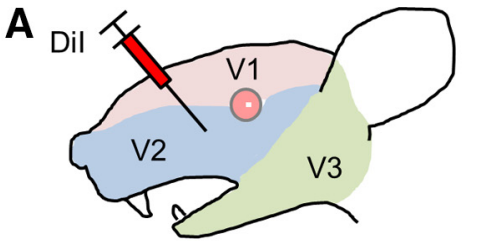

B

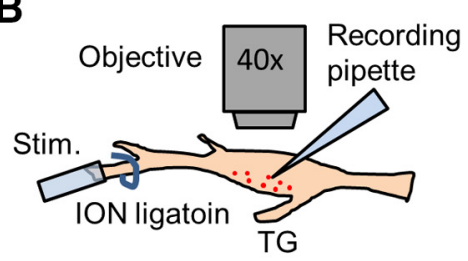

D

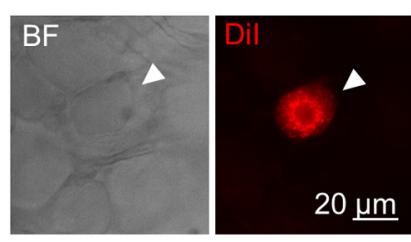

F

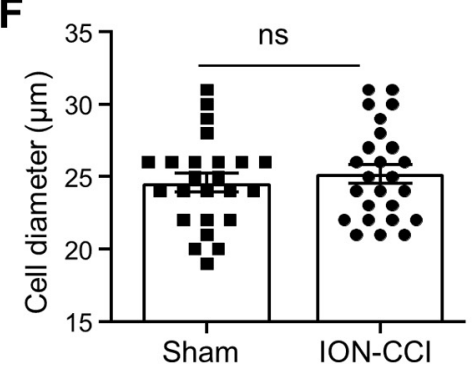

H

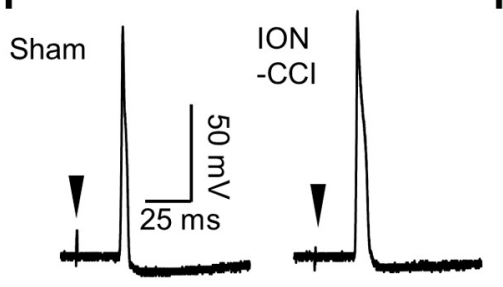

C

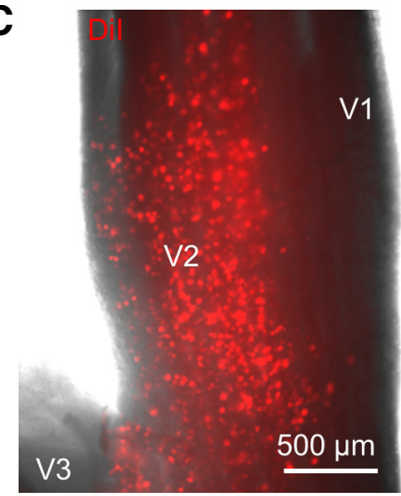

E

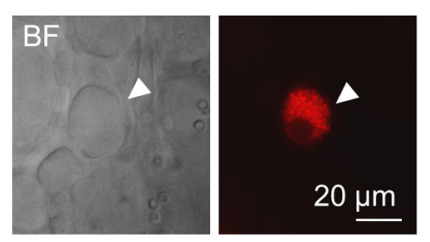

G

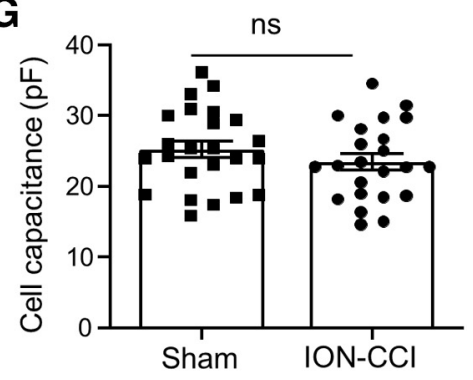

I

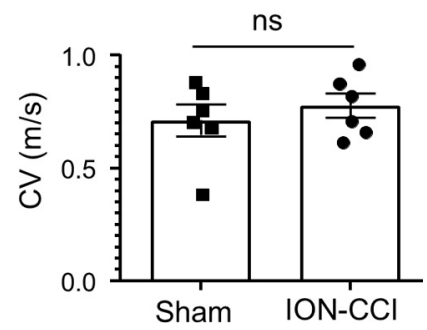

Figure 2. Patch-clamp recordings from small-sized V2 TG neurons innervating orofacial cutaneous regions of the sham and the ION-CCl rats. $A$, Illustration of intracutaneous injection of Dil in orofacial (V2) regions. The injections were performed in the sham or the $10 \mathrm{~N}-\mathrm{CCl}$ group 5-7 d before patch-clamp recording experiments. $\boldsymbol{B}$, Illustration of patch-clamp recordings from Dil-labeled orofacial cutaneous afferent neurons in the whole-mount ex vivo TG preparations. The relative positions of recording site, nerve ligation site, and stimulation site are indicated. C, Image captured under a fluorescent microscope with a $5 \times$ object shows the distribution of Dil-labeled neurons in the V2 region of a fresh TG. D, Bright field image (left) and fluorescent image (right; Dil) show in the same field a small-sized Dil-labeled V2 TG neuron (arrow indicated) in a whole-mount ex vivo TG preparation of the sham group. Patch-clamp recording was applied to the TG neuron. $\boldsymbol{E}$, Similar to $\boldsymbol{D}$ except the TG preparation was from the ION-CCl group. $\boldsymbol{F}, \mathbf{G}$, Diameters $(\boldsymbol{F})$ and membrane capacitance $(\boldsymbol{G})$ of the small-sized Dil-labeled V2 TG neurons in ex vivo TG preparation of the sham $(n=24)$ and the ION-CCl groups $(n=26)$ included in the patchclamp recording experiments. $\boldsymbol{H}$, A sample trace of an AP recorded from a small-sized Dil-labeled V2 TG neuron of the sham group (left) and another sample trace of an AP recorded from a small-sized Dil-labeled V2 TG neuron of the ION-CCl group. Arrow in each trace indicates stimulation artifact. $I$, Summary data of conduction velocities of APs propagated from peripheral stimulation sites on afferent nerve fibers to the recording sites on the somas of the small-sized Dil-labeled V2 TG neurons. Open bar, sham group $(n=6)$; closed bar, ION-Cl group $(n=6)$. Data represent mean \pm SEM; NS, no significant difference.

the Prolong Diamond Antifade Mountant medium (ThermoFisher Scientific). Slices were viewed under an upright fluorescent microscope (BX-43, Olympus) and images were captured in the TG V2 regions using a CCD camera (DP80, Olympus).
Semi-quantitative RT-PCR

Individual TGs were dissected out from the sham or the ION-CCI groups. Each TG was placed in a $2 \mathrm{ml}$ RNasefree centrifuge tube that contained $600 \mu \mathrm{l}$ ice-cold RNase-free lysis buffer with 1\% 2-mercaptoethanol. The TG in the tube was then homogenized using a Model 398 Tissue Tearor homogenizer (Biospec Products Bartlesville) at the speed of 30,000 rpm. Following a centrifuge at $21,000 \times g$ at $4^{\circ} \mathrm{C}$ for $15 \mathrm{~min}$, the supernatant was transferred to another $2 \mathrm{ml}$ RNase-free tube, and $600 \mu \mathrm{l}$ of $70 \%$ ethanol made with RNase-free water was added to the supernatant. Total RNAs were isolated using the PureLink RNA mini kit (ThermoFisher Scientific) following the manufacturer's instructions. Briefly, the mixture of the supernatant of tissue lysate and ethanol was passed through a nucleic acid binding column, centrifuged and sequentially washed with the buffers provided in the PureLink RNA mini kit, and then eluted in $30 \mu \mathrm{l}$ of RNase-free water. The concentration of RNAs of each sample was quantitated by the Qubit RNA HS Assay kit (ThermoFisher Scientific). The RNAs of each sample at an equal amount of $1.5 \mu \mathrm{g}$ were reverse transcribed with the iScript cDNA Synthesis kit (Bio-Rad) according to manufacturer's instructions, under the following thermal cycler conditions: $5 \mathrm{~min}$ at $25^{\circ} \mathrm{C}, 40 \mathrm{~min}$ at $42^{\circ} \mathrm{C}, 5 \mathrm{~min}$ at $85^{\circ} \mathrm{C}$, and held for $5 \mathrm{~min}$ at $4^{\circ} \mathrm{C}$.

Primers were designed with NCBI/Primer-BLAST tool and BLASTed against the rat Refseq_mRNA database to test for specificity and contained at least one sequence that crossed an exon-exon boundary to avoid amplification of genomic DNA. The specificity of all primers was confirmed using gel electrophoresis. PCR primers were: Kv4.3 (accession number AB003587.1) forward CTCCAATGCCTA CCTGCACA, reverse GGTGGAGGTTCGTACAGACA; product: $202 \mathrm{bp}$. GAPDH (NM017008.4) forward ACT TTGGCATCGTGGAAGGG, and reverse ACTTGGCA GGTTTCTCCAGG; product: 244 bp. RT-PCR was performed in a $25-\mu$ l reaction solution with the One Tag Hot Start DNA polymerase (New England Biolabs) and dNTP mixture (ThermoFisher Scientific) on an Applied Biosystems 2720 Thermal Cycler (ThermoFisher Scientific) with an initial $94^{\circ} \mathrm{C}$ Taq activation step for $5 \mathrm{~min}$, followed by 30 cycles of $94^{\circ} \mathrm{C}$ for $30 \mathrm{~s}, 60^{\circ} \mathrm{C}$ for $30 \mathrm{~s}$, and $72^{\circ} \mathrm{C}$ step for $30 \mathrm{~s}$, followed by one cycle at $72^{\circ} \mathrm{C}$ for $30 \mathrm{~s}$ and one cycle at $68^{\circ} \mathrm{C}$ for $5 \mathrm{~min}$. Following the cycling, samples were evaluated by gel electrophoresis on a $0.8-2 \%$ agarose gel and imaged using the ChemiDoc- $\mathrm{It}^{2}$ Imagers (Ultra-Violet Products Ltd.).

\section{Data analysis}

In orofacial operant behavioral tests, the dependent variables of the rat's behavior were total contact time and total contact numbers. For the data of electrophysiological recordings, membrane parameters and voltage-activated currents were analyzed using Clampfit 10 software. For the immunostaining, fluorescent images of TG V2 regions were acquired using the Olympus CellSens imaging software (Olympus), and Kv4.3-immunopositive and Kv4.3-immunonegative neurons in TG V2 regions were then identified from the fluorescent images based on their relative fluorescent intensity. A cell with its fluorescent intensity signal to background noise ratio of $\geq 3: 1$ is considered to be Kv4.3-immunopositive, and the ratio of $<3: 1$ is considered to be Kv4.3-immunonegative. The percent of Kv4.3-immunoreactivity (ir)-positive neurons was then calculated. The quantification of the immunohistochemistry results was not blinded as to whether a sample was from an ION-CCI animal or a control animal. However, the quantification was performed by a lab 
personnel who did not know whether Kv4.3 expression in ION-CCI was expected to be increased, decreased, or no change. For RT-PCR experiments, RT-PCR product band density of Kv4.3 mRNAs was measured by densitometry with ChemiDoc- $\mathrm{It}^{2}$ Imagers (Ultra-Violet Products Ltd.) and expressed as relative density to GAPDH RT-PCR product. Unless otherwise indicated, all data are reported as mean \pm SEM of $n$ independent observations. Statistical comparisons were made using GraphPad Prism 8 software with ${ }^{*} p<0.05$, ${ }^{* *} p<0.01$, and ${ }^{* * *} p<$ 0.001 , Student's $t$ test or two-way ANOVA with Bonferroni post hoc tests for multiple groups.

\section{Results}

ION-CCI induces orofacial behavioral cold hypersensitivity

We created ION-CCI by infraorbital nerve ligation to induce trigeminal neuropathic pain in orofacial regions. The presence of neuropathic pain manifested with cold hypersensitivity in orofacial regions was demonstrated by the use of the orofacial operant test with thermal module set at $5^{\circ}$ C (Fig. 1). The orofacial operant test is designed to create a conflictive condition for animals to obtain the reward of sweetened milk while receiving cold stimulation. The operant test measures pain rather than a simple nociceptive reflex (Cha et al., 2012). Although the temperature of $5^{\circ} \mathrm{C}$ was a noxious cold stimulus, sham animals were still willing to rest their cheeks on the thermal module and spend time to drink milk (Fig. $1 A$ ). In contrast, ION-CCI rats avoided to have prolonged contacts to the cooling thermal module with their cheeks, and sometimes they had some unusual drinking postures such as turning their heads upside down (Fig. 1B) or guarded their orofacial regions with their front paws (Fig. $1 C$ ). We quantitatively measured orofacial operant behaviors using the total contact time and contact numbers in the 10-min test session. The total contact time was $188.1 \pm 18.2 \mathrm{~s}(n=6)$ in the sham group (Fig. $1 D, F)$, significantly decreased to $49.9 \pm 17.3 \mathrm{~s}(n=6)$ in the ION-CCI group (Fig. $1 E, F)$. The total contact numbers were not significantly different between the sham group $(n=6)$ and the ION-CCI group (Fig. 1G). Similar to total contact time, the mean contact time was significantly shorter in the ION-CCI group $(1.8 \pm 0.2 \mathrm{~s}, n=6)$ than in the sham group ( $4.8 \pm 0.6 \mathrm{~s}$, $n=6$; Fig. $1 H)$. The above experiments were performed in six ION-CCI rats and six sham rats, and all six ION-CCI displayed exacerbated orofacial pain behaviors manifested with coldhypersensitivity during the orofacial operant test. These results demonstrate that our ION-CCI model reliably creates trigeminal neuropathic pain with cold hypersensitivity in orofacial regions.

ION-CCI induces neuronal hyperexcitability in small-sized V2 TG neurons that innervates orofacial cutaneous region Orofacial regions are innervated by maxillary nerves, the V2 branches of the TGs (V2 TGs). To identify V2 TG neurons innervating orofacial cutaneous regions for patch-clamp recordings, we retrogradely labeled these V2 TG neurons by intracutaneous injections of the fluorescent dye DiI in the cheeks of the sham and the ION-CCI rats (Fig. 2A). One week after the DiI injections, TGs with attached nerve bundles were dissected out and ex vivo TG preparations were then made for patchclamp recordings (Fig. $2 B$ ). Under the fluorescent microscope, many DiI-labeled TG neurons could be observed in the ex vivo TG preparations and they were distributed in the V2 TG regions (Fig. $2 B, C$ ). Since we were interested in nociceptive type of orofacial cutaneous afferent neurons, we only applied patch-clamp recordings to the small-sized DiI-labeled V2 TG neurons in both the sham and the ION-CCI groups (Fig. $2 B, D, E$ ). In this set of experiments, the small-sized DiI-labeled V2 TG neurons selected for patch-clamp recordings had mean diameters of $24.6 \pm 0.6$ $\mu \mathrm{m}(n=24)$ and $25.6 \pm 0.7 \mu \mathrm{m}(n=26)$ in the sham group and the ION-CCI group, respectively (Fig. $2 F$ ). The whole-cell membrane capacitance was $25.2 \pm 1.2 \mathrm{pF}(n=24)$ and $25.9 \pm 1.7 \mathrm{pF}$ $(n=26$; Fig. $2 G)$ in the sham group and the ION-CCI group, respectively. There were no significant differences in cell sizes and membrane capacitance between the selected small-sized DiIlabeled V2 TG neurons of the sham and the ION-CCI groups (Fig. 2F,G, $p>0.05$ ). We determined whether the small-sized DiI-labeled V2 TG neurons were primarily arisen from orofacial cutaneous C-afferent fibers by measuring their conduction 
A

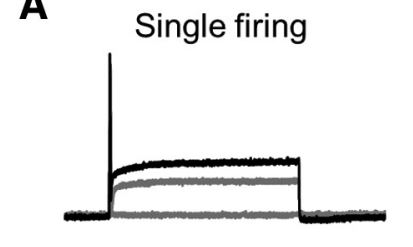

B

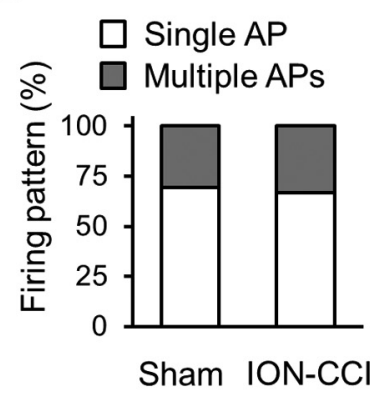

Multiple firing

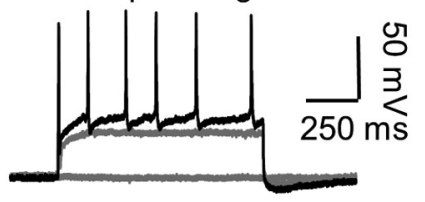

C

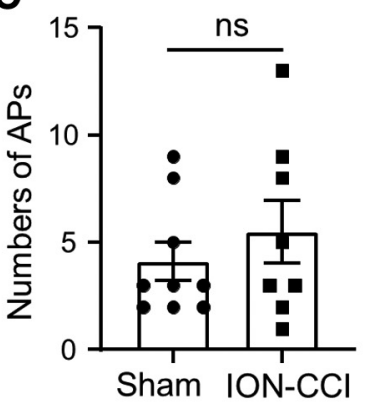

D

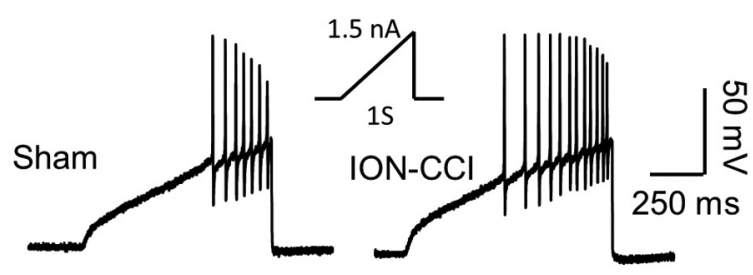

E

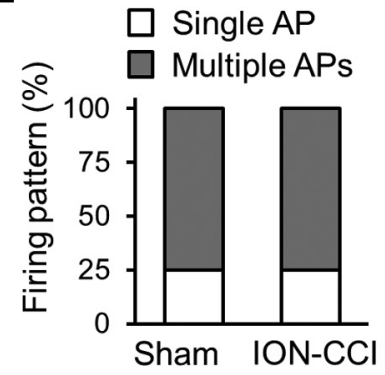

F

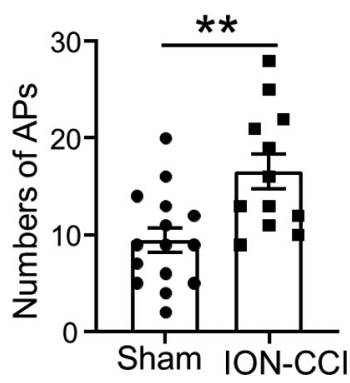

Figure 4. AP firing numbers in small-sized V2 TG neurons of sham group and ION-CCl group. A, Two sets of sample traces show two types of AP firing patterns recorded from small-sized Dil-labeled V2 TG neurons, the single AP firing pattern (left) and the multiple AP firing pattern (right). The two sets of traces were from two small-sized Dil-labeled V2 TG neurons of the sham group. $\boldsymbol{B}$, Percentages of small-sized Dil-labeled V2 TG neurons showing single AP firing pattern (open bars) and multiple AP firing pattern (closed bars) in the sham group ( $n=24)$ and the ION-CCl group $(n=26)$. C, Numbers of APs in small-sized Dil-labeled V2 TG neurons with multiple AP firing in the sham ( $n=24$, closed circles) and the ION-CCI groups ( $n=26$, closed squares). In A-C, APs were elicited by stepwise currents of 2 times of AP rheobases. D, Sample traces show APs evoked by ramp currents injected via recording electrodes in a small-sized Dil-labeled V2 TG neuron of the sham group (left) and the smallsized Dil-labeled V2 TG neuron of ION-CCl group (right). Inset, the ramp current amplitude and duration. $\boldsymbol{E}$, Percentages of small-sized V2 TG neurons showing single AP firing pattern (open bars) and multiple AP firing pattern (closed bars) in the sham group $(n=20)$ and the ION-CCI group $(n=16)$. $\boldsymbol{F}$, Numbers of APs of small-sized Dil-labeled V2 TG neurons with multiple AP firing in the sham group ( $n=20$, closed circles) and the ION-CCl group ( $n=16$, closed squares). In $\boldsymbol{E}, \boldsymbol{F}, \mathrm{APs}$ were elicited by ramp currents. Data represent mean $\pm \mathrm{SEM}$; NS, no significant difference; $* * p<0.01$.

velocity. In this set of experiments, APs were recorded under the current-clamp configuration from randomly sampled smallsized DiI-labeled V2 TG neurons while electrical stimulation was applied to the peripheral sites of trigeminal nerve bundles (Fig. 2B,H). In each small-sized DiI-labeled V2 TG neuron recorded, APs appeared after long latencies following the peripheral electrical stimulation, and each AP displayed a shoulder in

A

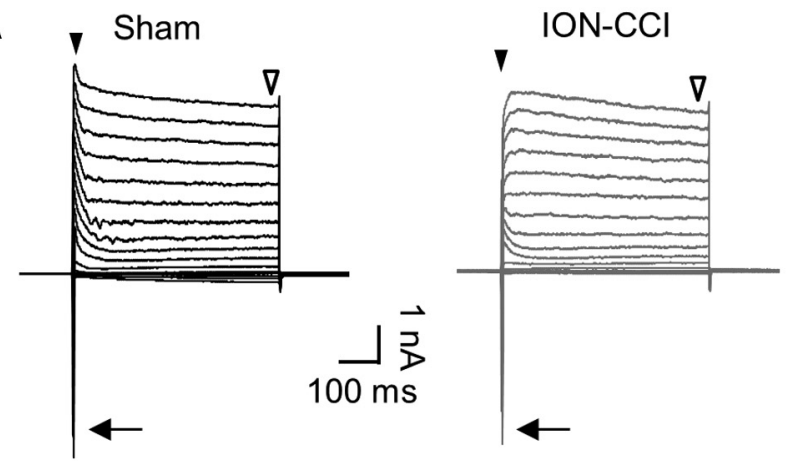

B Initial component $(\mathrm{pA} / \mathrm{pF})$
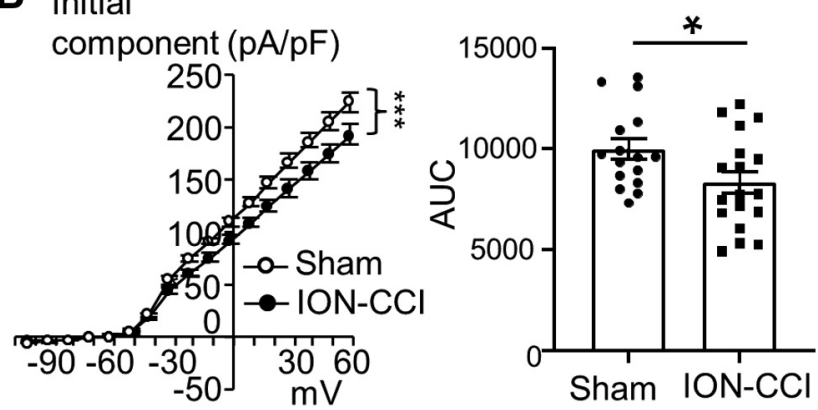

C

Late
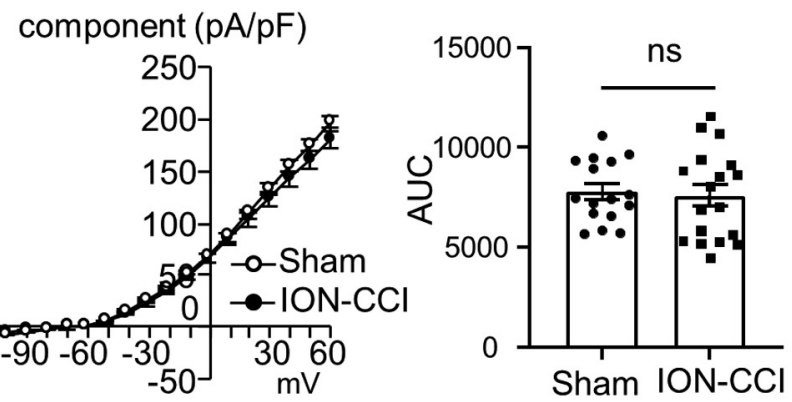

Figure 5. $\quad 10 \mathrm{~N}-\mathrm{CCl}$ results in a decrease of voltage-activated outward currents in smallsized V2 TG neurons that innervate orofacial cutaneous regions. $\boldsymbol{A}$, Two sets of sample traces of voltage-activated currents recorded from a small-sized Dil-labeled V2 TG neuron of the sham group (left) and a small-sized Dil-labeled V2 TG neuron of the ION-CCl group. In each panel, arrow indicates the transient inward currents, closed arrowhead indicates initial components of the outward currents, and open arrowhead indicates late components of the outward currents. B, left panel, I-V curves of initial components of the outward currents recorded in small-sized Dil-labeled V2 TG neurons of the sham (open circles, $n=24$ ) and the ION-CCl groups (closed circles, $n=26$ ). Right panel, AUC from the voltages of 0 to $58 \mathrm{mV}$ for the sham group ( $n=24$, closed circles) and the $10 \mathrm{~N}-\mathrm{CCl}$ group $(n=26$, closed squares). $\boldsymbol{C}$, Similar to $\boldsymbol{B}$ except graphs are late components of the outward currents of the sham group $(n=24)$ and the ION-CCl group $(n=26)$. Data represent mean \pm SEM; NS, no significant difference; ${ }^{*} p<0.05$, ${ }^{* * *} p<0.001$.

its repolarization phase (Fig. $2 H$ ). The conduction velocities of action potentials were $0.71 \pm 0.04 \mathrm{~m} / \mathrm{s}(n=6)$ and $0.78 \pm 0.03 \mathrm{~m} /$ $\mathrm{s}(n=6)$ for the sham group and the ION-CCI group, respectively, and were not significantly different between the two groups $(p>0.05 ;$ Fig. $2 I)$. The conduction velocity of both groups fell into the range of the speed of $\mathrm{C}$-afferent fibers, suggesting that they were orofacial cutaneous $\mathrm{C}$-afferent neurons.

We determined whether ION-CCI resulted in an increase in the excitability of the small-sized V2 TG neurons that innervated orofacial cutaneous regions. In this set of experiments, APs were evoked by stepwise currents injected through patch-clamp 
electrodes into the small-sized DiI-labeled V2 TG neurons. Similar to APs evoked by peripheral stimulation (Fig. 2H), APs evoked by the stepwise currents also displayed shoulders in their repolarization phases in the small-sized DiI-labeled V2 TG neurons of both the sham and the ION-CCI groups (Fig. 3A, $B)$. We characterized membrane and AP properties of both the sham and the ION-CCI groups (Fig. 3C$H)$. Membrane input resistance in the ION-CCI group (283.9 $\pm 30.3 \mathrm{M} \Omega, n=26$; Fig. $3 C$ ) appeared to be higher than that in the sham group $(227.7 \pm 23.1$ $\mathrm{M} \Omega, n=24$; Fig. $3 C$ ), although the difference did not reach a statistical significance $(p=0.06)$. Resting membrane potentials were $-72.5 \pm 1.2 \mathrm{mV}$ in the small-sized DiI-labeled V2 TG neurons of the sham group $(n=24)$, and were significantly depolarized to $-69.5 \pm 1.1 \mathrm{mV}(n=26, p<0.05)$ in the small-sized DiI-labeled V2 TG neurons of the ION-CCI group (Fig. 3D). Rheobase for AP initiation was $352.2 \pm 24.3 \mathrm{pA}(n=24)$ in the small-sized DiI-labeled V2 TG neurons of the sham group, and was significantly reduced to $235.4 \pm 21.8 \mathrm{pA} \quad(n=26$, $p<0.01)$ in the small-sized DiI-labeled V2 TG neurons of the ION-CCI group (Fig. 3E). AP widths were $2.4 \pm 0.2 \mathrm{~ms}(n=24)$ in small-sized DiI-labeled TG neurons of sham group, and were significantly broadened to $3.2 \pm 0.2 \mathrm{~ms}(n=26, p<0.05)$ in the small-sized DiI-labeled TG neurons of ION-CCI group (Fig. 3F). AP thresholds (Fig. 3G) and AP amplitudes (Fig. $3 H$ ) were not found to be significantly different between the sham and the ION-CCI groups.

We determined whether AP firing numbers and firing patterns in the small-sized DiI-labeled V2 TG neurons were different between the sham and the ION-CCI groups. In this set of experiments, we first examined APs elicited by stepwise currents. In both the sham and the ION-CCI groups, the majority of small-sized DiI-labeled V2 TG neurons only fired single APs but a small number of small-sized DiI-labeled V2 TG neurons fired multiple APs in response to the stepwise current injections (Fig. $4 A, B)$. As shown in Figure $4 B$, in the sham group, $\sim 68 \%$ of small-sized DiI-labeled V2 TG neurons fired a single AP and the remaining $32 \%$ of the neurons fired multiple APs (total 24 cells; Fig. $4 B$ ). In the ION-CCI group, $67 \%$ of small-sized DiI-labeled V2 TG neurons fired a single AP and the remaining $33 \%$ of the neurons fired multiple APs (total 26 cells; Fig. 4B). There were no significant differences in the AP firing patterns between the sham and the ION-CCI group $(p>0.05)$. For the neurons firing multiple APs, the numbers of APs at $2 \times$ rheobase were not significantly different between the sham and the ION-CCI groups (Fig. 4C). We further tested the above neurons to determine their AP firing patterns and firing numbers in responses to the injections of ramp currents (Fig. 4D). The majority of the small-sized DiI-labeled V2 TG neurons fired multiple APs in responsible to the ramp currents (Fig. $4 E$ ). As show in Figure $4 E$, in the sham group, $80 \%$ of neurons fired multiple APs and remaining $20 \%$ of the neurons fired a single AP (total 20 cells). In the ION-CCI group, $75 \%$ of the neurons fired multiple APs and remaining $25 \%$ of the neurons fired a single AP (total 16 cells; Fig. $4 E$ ). There were no significant differences in the firing patterns between the sham and the ION-CCI group. However, for the small-sized DiI-labeled V2 TG neurons firing multiple APs with the injections of ramp currents, the numbers of APs were
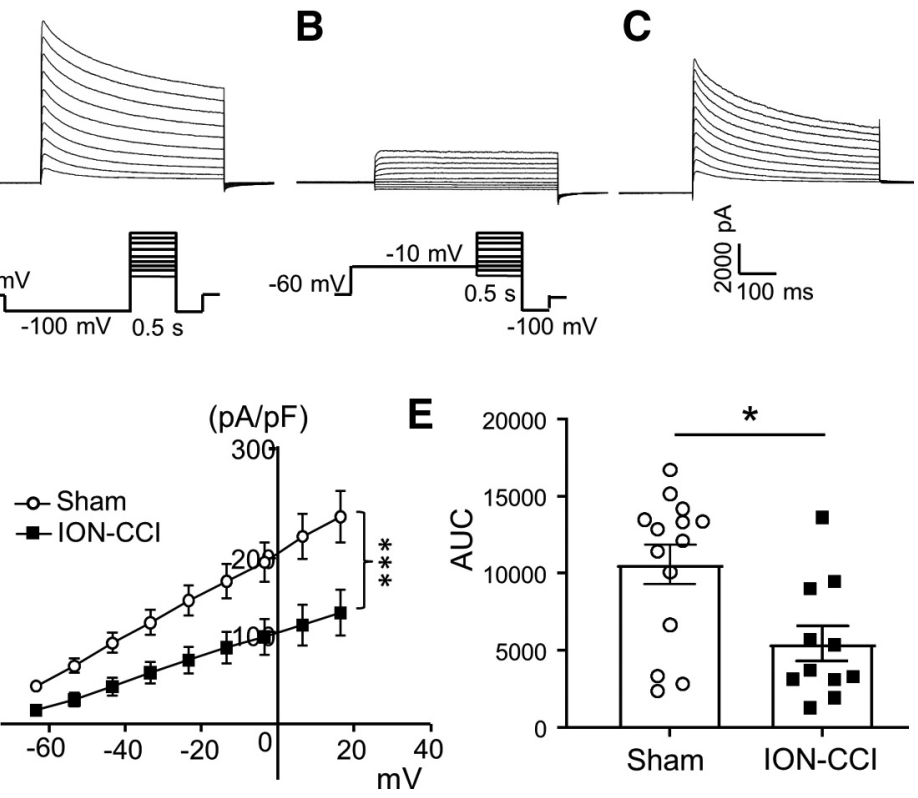

Figure 6. $I O N-C C$ results in a reduction of IA currents in small-sized V2 TG neurons. $\boldsymbol{A}-\boldsymbol{C}$, Sample traces ward currents $(\boldsymbol{C})$. IA currents were obtained by the subtraction of total outward currents $(\boldsymbol{B})$ from noncommand voltages for generating total outward currents in $\boldsymbol{A}$ and rents recorded from small-sized V2 TG neurons of the sham group ( $n=14$, open circles) and the ION-CC Summary data of the AUC in the sham group ( $n=14$, open circles) and the ION-CCl group $(n=11$, closed significantly increased following ION-CCI (Fig. 4F). The AP numbers were $9.5 \pm 1.3 \mathrm{APs} / \mathrm{s}(n=15)$ in the sham group, and significantly increased to $16.6 \pm 1.8 \mathrm{APs} / \mathrm{s}(n=12, p<0.01)$ in the ION-CCI group (Fig. 4F)

\section{ION-CCI results in a reduction of IA currents in small-sized V2 TG neurons}

To explore ion channel mechanisms underlying the changes of membrane and AP properties of orofacial cutaneous $\mathrm{C}$-afferent neurons following ION-CCI, we examined voltage-activated currents in both the sham and the ION-CCI groups. Under the voltage-clamp configuration, voltage-activated currents were elicited from the small-sized DiI-labeled V2 TG neurons by a series of depolarizing voltage steps. As shown in Figure 5A, depolarizing voltage steps evoked inward currents as well as outward currents in the small-sized DiI-labeled V2 TG neurons of both the sham and the ION-CCI groups. The inward current components were transient, consistent with the currents mediated by voltage-gated sodium channels (Fig. 5A). The outward currents consisted of two phases, the initial component (Fig. $5 A, B$ ) and the later component (Fig. $5 A, C$ ). The initial component is mainly composed of the IA, and the late component mainly consisted of non-inactivating voltage-activated $\mathrm{K}^{+}$current (IK; Viatchenko-Karpinski et al., 2018). Current-voltage relationship (I-V curve) of the initial components in the small-sized DiI-labeled V2 TG neurons showed that these currents were smaller in the ION-CCI group than in the sham group (Fig. 5B). For example, the initial components evoked by the voltage step from -72 to $28 \mathrm{mV}$ were $141.2 \pm 7.2 \mathrm{pA} / \mathrm{pF}(n=23)$ in the small-sized DiI-labeled V2 TG neurons of ION-CCI group, and was significantly smaller than the initial components of $170 \pm 10.6 \mathrm{pA} / \mathrm{pF}(n=24, p<0.05)$ in the small-sized DiI-labeled V2 TG neurons of the sham group. 
A

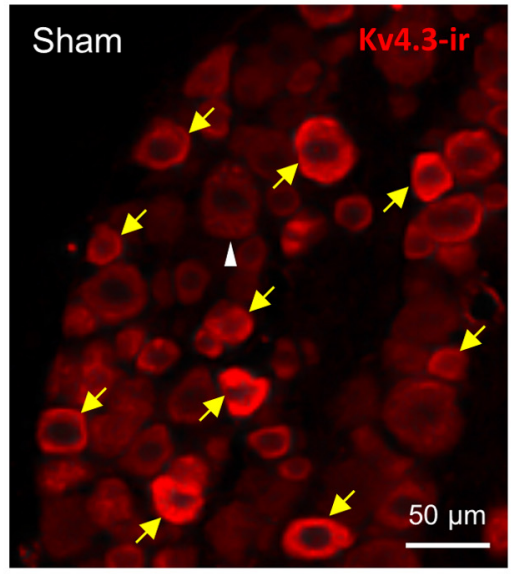

B
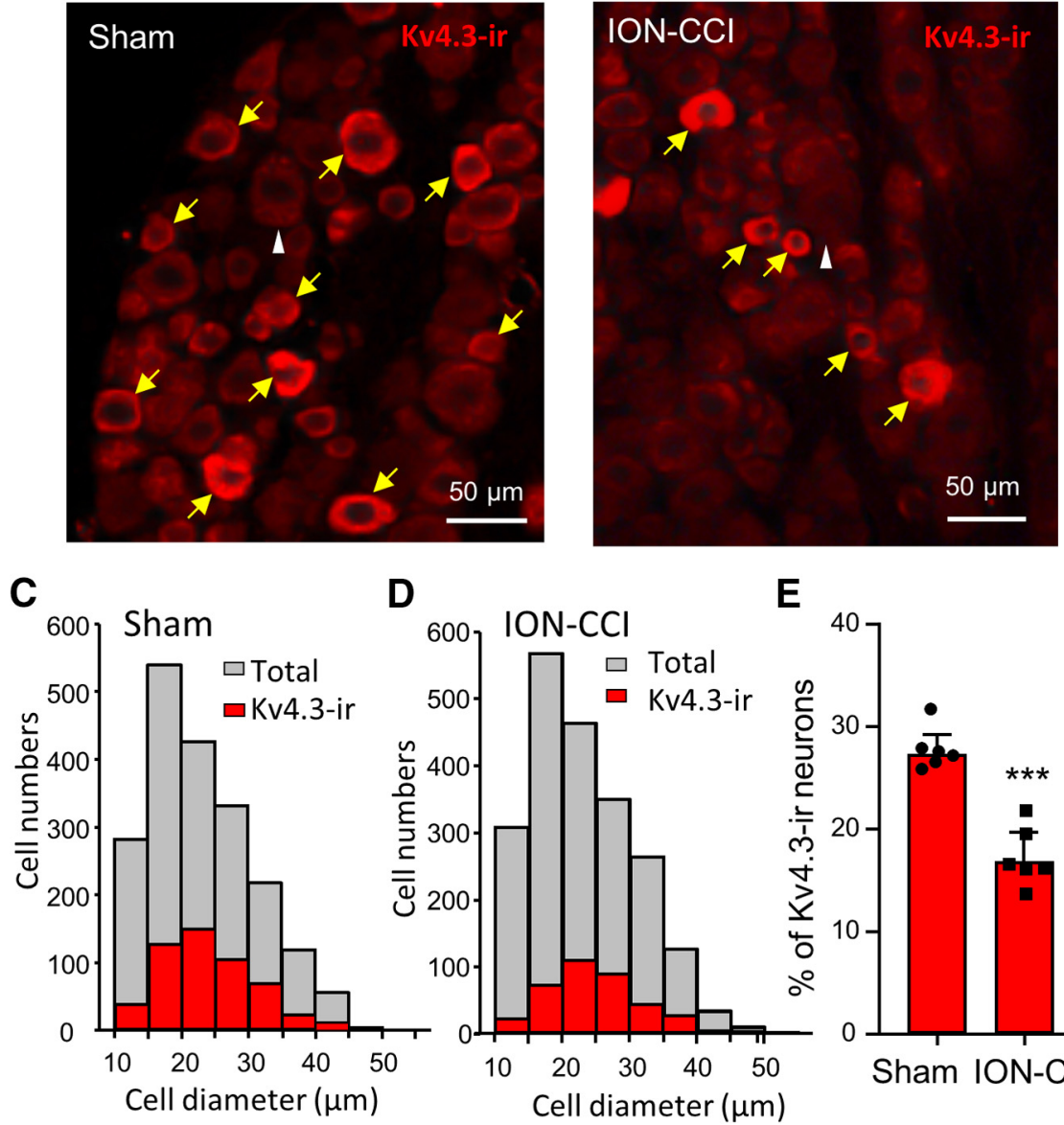

$\mathbf{F}$

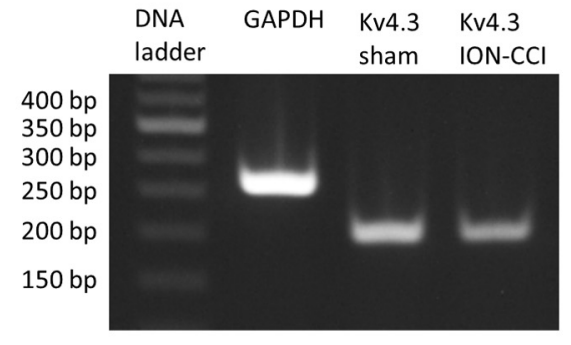

E

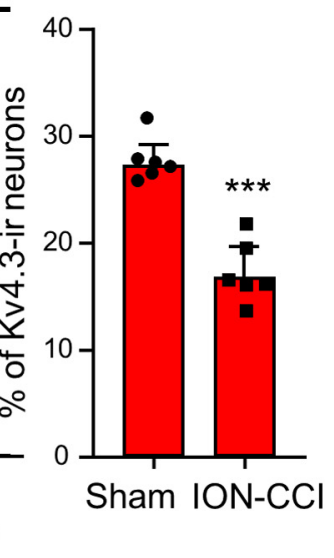

G

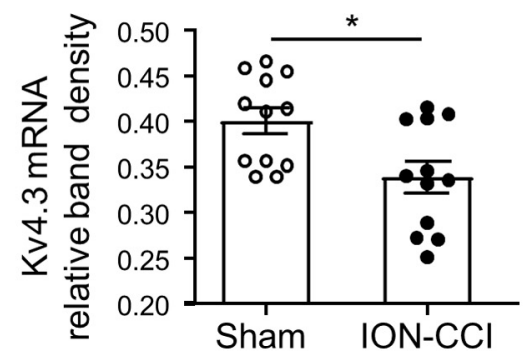

Figure 7. $\quad \mathrm{ION}-\mathrm{CCl}$ results in downregulation of Kv4.3 expression in small-sized V2 TG neurons. $\boldsymbol{A}$, Image shows Kv4.3-ir in the V2 TG section from the sham group. $\boldsymbol{B}$, Similar to $\boldsymbol{A}$ except the V2 TG section was from the ION-CCl group. In $\boldsymbol{A}, \boldsymbol{B}$, a white arrowhead indicates a large TG neuron that is Kv4.3 negative, and yellow arrows indicate Kv4.3-ir-positive TG neurons. $\boldsymbol{C}$, Histograms of Kv4.3-ir-positive (red, 546 cells) and total V2 TG neurons (gray, 1975 cells, 6 rats) in the sham group. D, Histograms of Kv4.3-ir-positive (red, $n=363$ cells) and total V2 TG neurons (gray, 2132 cells, 6 rats) in the ION-CCI group). $\boldsymbol{E}$, Summary data of the percent of Kv4.3 V2 TG neurons in the sham group ( $n=1975$ cells from 6 rats) and the ION-CCl group ( $n=2132$ cells from 6 rats). $\boldsymbol{F}$, Agarose gel photograph shows RT-PCR products of Kv4.3 mRNAs from a TG of shame group (lane 3) and a TG of ION-CCl group (lane 4). Lane 1, 50-bp DNA ladder; lane 2, GAPDH RT-PCR product. G, Summary data of RT-PCR products of KV4.3 mRNAs in individual TGs of sham group (12 TGs from 6 rats) and of ION-CCl group (12 TGs from 6 rats). RT-PCR product band density was measured by densitometry and expressed as relative density to GAPDH RT-PCR product. Data represent mean $\pm \mathrm{SEM} ; * p<0.05, * * * p<0.001$.

Comparison was also made using the areas under the I-V curves of the initial components and showed significantly lower values in the ION-CCI group $(n=26)$ than in the sham group $(n=24$; Fig. $5 B$ ). In contrast, the late components in the small-sized DiIlabeled V2 TG neurons showed no significant difference between the sham $(n=24)$ and the ION-CCI groups $(n=26$; Fig. $5 C)$.

To confirm that IA currents in the small-sized V2 TG were reduced following ION-CCI, we isolated IA currents from the total outward currents and determined IA currents in both the sham and the ION-CCI groups (Fig. 6). In this set of experiments, the V2 TG neurons of the sham and the ION-CCI groups were recorded under voltage-clamp mode and IA currents were isolated by an electrophysiological approach. Figure $6 A-C$ shows the experimental protocol and sample traces that demonstrate the isolation of IA currents recorded in a smallsized V2 TG neuron. I-V relationship (Fig. $6 D$ ) shows that the isolated IA currents in the ION-CCI group $(n=11)$ were smaller than those in the sham group $(n=14)$. Comparison was also made using the areas under the $\mathrm{I}-\mathrm{V}$ curve (AUCs) of the isolated IA currents between the sham $(n=14)$ and the IONCCI groups $(n=11)$, and the AUC values were significant smaller in the ION-CCI group than in the sham group $(p<0.05$; Fig. $6 E)$.

\section{ION-CCI leads to downregulation of Kv4.3 channel expression in small- sized V2 TG neurons}

We determined whether the expression of Kv4.3 channels was downregulated in small-sized V2 TG neurons following IONCCI since Kv4.3 channels can mediate IA currents (Zemel et al., 2018). Kv4.3 channels were found to be mainly expressed in small-sized V2 TG neurons in both the sham and the ION-CCI groups (Fig. 7A$D$ ). The mean diameters of Kv4.3-ir-positive V2 TG neurons were $24.1 \pm 0.3 \mu \mathrm{m}$ ( $n=546$ positive neurons) in the sham group and were $24.7 \pm 0.3 \mu \mathrm{m} \quad(n=363$ positive neurons) in the ION-CCI group, and the sizes of the two groups were not significantly different. We compared percentage of Kv4.3-ir-positive V2 TG neurons between the sham group and the ION-CCI group. In the sham group with a total of 1975 V2 TG neurons examined, $\sim 27 \%$ $(27.3 \pm 0.8 \%, 18$ TG sections from 6 rats, $n=6$; Fig. $7 C, E)$ of them were Kv4.3-ir-positive. In the ION-CCI group with a total of 2132 V2 TG neurons examined, Kv4.3ir-positive V2 TG neurons were $\sim 17 \%$ $(16.9 \pm 1.1 \%, 18$ TG sections of 6 rats, $n=6$; Fig. $7 D, E)$, significantly less than that of the sham group $(p<0.001)$. We also examined total numbers of $\mathrm{V} 2 \mathrm{TG}$ neurons in both the sham group and the ION-CCI group to see whether there was a net loss of the V2 TG neurons after ION-CCI. In the sham group the averaged numbers of V2 TG neurons in each field of a V2 section were $101.4 \pm 3.3$ (18 sections of 6 rats, $n=18$, not graphically illustrated), not significantly different from those of the sham group (102.2 \pm 5.3 cells, 18 section of 6 rats, $n=18$, not graphically illustrated). Consistent with the immunostaining results, downregulation of Kv4.3 mRNA expression in TG 
neurons following ION-CCI was shown using a semi-quantitative RT-PCR approach (Fig. $7 F, G$ ). As shown in Figure $7 F, G$, the relative band density of RT-PCR products for Kv4.3 mRNAs was significantly lower in the ION-CCI group $(n=12, p<0.05)$ than in the sham group $(n=12)$.

Phrixotoxin-2 suppresses IA currents in small-sized V2 TG neurons and induces orofacial behavioral cold

hypersensitivity

To determine whether Kv4.3 channels play a significant role in mediating IA currents in small-sized V2 TG neurons, we tested whether IA currents in these neurons could be significantly inhibited by phrixotoxin-2, a blocker of Kv4.3 channels (Diochot et al., 1999). In this set of experiments, the inhibition was defined as a reduction of IA currents by $>15 \%$ following phrixotoxin2 applications based on data clustering (Fig. $8 A-C$ ). We found that IA currents in the majority of small-sized V2 TG neurons $(60 \%)$ were inhibited by $70 \mathrm{~nm}$ phrixotoxin-2 (Fig. $8 A, C, D$ ). In this group of TG neurons, the IA currents were $55.8 \pm 12.1 \mathrm{pA} / \mathrm{pF}$ in the control and significantly reduced to $36.4 \pm 8.3 \mathrm{pA} / \mathrm{pF}$ ( $n=$ $12, p<0.01$ ) following the bath application of phrixotoxin-2 (Fig. 8D). There were also many small-sized V2 TG neurons (40\%) whose IA currents were not inhibited by phrixotoxin-2 (Fig. $8 B-D$ ). In this phrixotoxin-2-insensitive group, the IA currents were $97.8 \pm 25.5 \mathrm{pA} / \mathrm{pF}$ in the control and were not significantly changed (98.7 \pm $25.1 \mathrm{pA} / \mathrm{pF}, n=8$ ) following the bath application of phrixotoxin-2 (Fig. 8D). Differences in phrixotoxin-2 sensitivity were also shown by the I-V curves for both phrixotoxin-2insenstive (Fig. $8 E$ ) and phrixotoxin-2-sensitive (Fig. $8 F$ ) neurons.

We next determined whether administration of phrixotoxin-2 at peripheral sites may affect orofacial cold sensitivity. In this set of experiments, normal rats were used and they were randomly divided into two groups, the control group for which vehicle (PBS saline) were bilaterally injected ( $50 \mu \mathrm{l}$; intracutaneous) in orofacial regions, and the phrixotoxin-2-injected group for which phrixotoxin-2 $(2 \mu \mathrm{M}, 50 \mu \mathrm{l}$, intracutaneous) were bilaterally injected in orofacial regions. Orofacial cold sensitivity was then determined using the orofacial operant test. As shown in Figure 9, when orofacial operant tests were performed with thermal module set at $5^{\circ} \mathrm{C}$, animals that were injected with phrixotoxin-2 showed significant decreases in the total contact time (Fig. 9A,B, $n=11$ ) and total contact numbers (Fig. 9A,C, $n=11$ ) in comparison
A

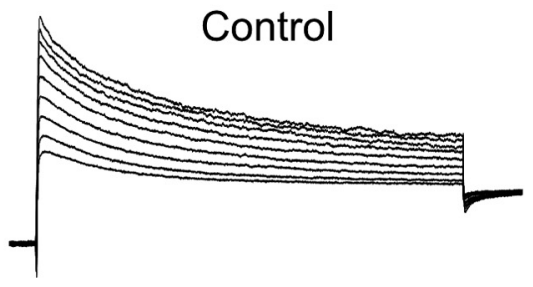

Phrixotoxin-2

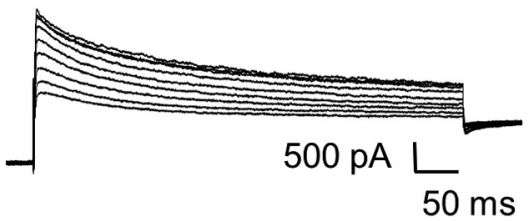

B

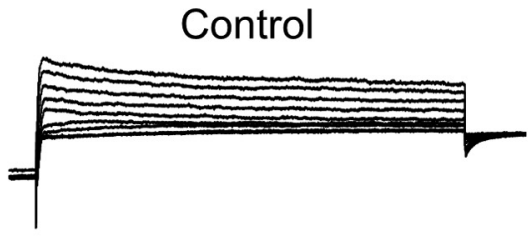

Phrixotoxin-2

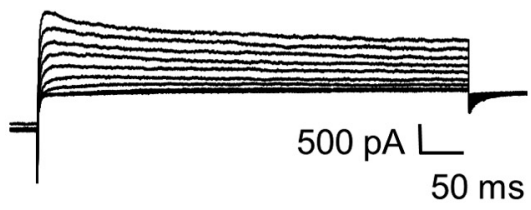

C

D Phrixotoxin-2 -sensitive

Phrixotoxin-2 -insensitive
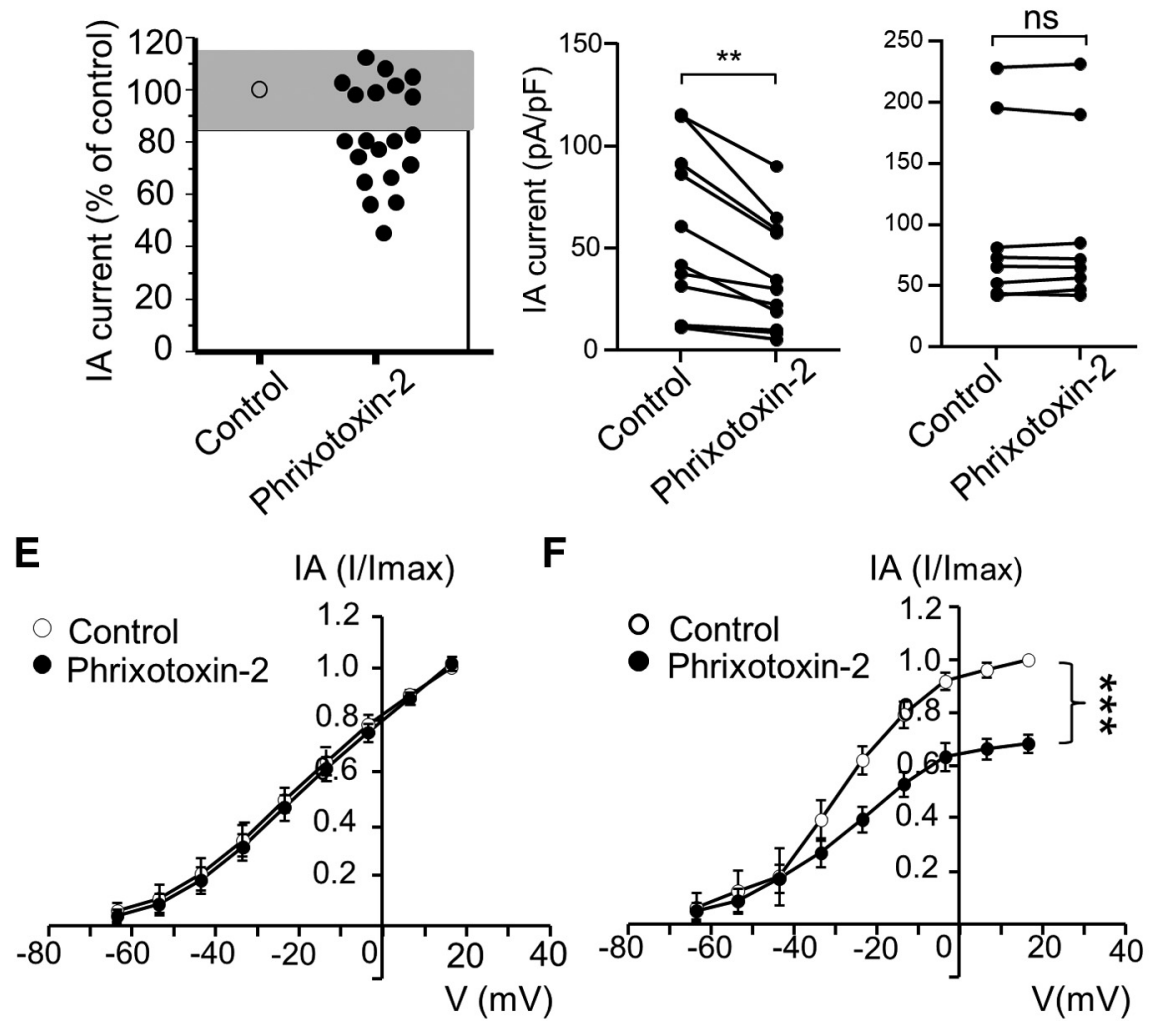

Figure 8. Kv4.3 blocker phrixotoxin-2 inhibits IA currents in small-sized V2 TG neurons. $A$, Sample traces show isolated IA currents in a small-sized V2 TG neuron before (control, left) and following the application of $70 \mathrm{~nm}$ phrixotoxin-2 (right). B, Sample traces show isolated IA currents in another small-sized V2 TG neuron before (control, left) and following the application of $70 \mathrm{~nm}$ phrixotoxin-2 (right). C, Graph shows changes of IA currents following the application of phrixotoxin-2. IA currents following phrixotoxin-2 were scaled to control values (\% of control). Cells whose IA currents showed changes less than $\pm 15 \%$ (gray area) were considered to be phrixotoxin-2-insensitive $(n=8)$; cells whose IA currents showed the reduction $>15 \%$ (white area) were considered to be phrixotoxin2-sensitive $(n=12)$. D, Pooled data of IA currents before (control) and following the bath application of $70 \mathrm{~nm}$ phrixotoxin-2. Left panel, Recordings from phrixotoxin-2-sensitive $(n=12)$ small-sized V2 TG neurons. Right panel, Recordings from phrixotoxin-2-insensitive $(n=8)$ small-sized V2 TG neurons. In both $\boldsymbol{C}, \boldsymbol{D}$, IA currents were evoked by a voltage step from -63.6 to $6.4 \mathrm{mV}$, and their mean values in the control and following the phrixotoxin-2 application were used for the statistical comparison. $\boldsymbol{E}, \boldsymbol{F}, \mathrm{I}-\mathrm{V}$ curves of isolated IA currents before (control, open circles) and following the application of phrixotoxin-2 (closed circles) in the phrixotoxin-2-insenstive group (left, $n=8$ ) and phrixotoxin-2-sensitive group (right, $n=12$ ). In $E, F$, IA current of each voltage point was normalized to the maximal current in control, and areas under the curves in the control and following the phrixotoxin-2 application were used for the statistical comparison. Data represent mean $\pm S E M$; NS, no significant difference; $* * p<$ $0.01, * * * p<0.001$ 
A

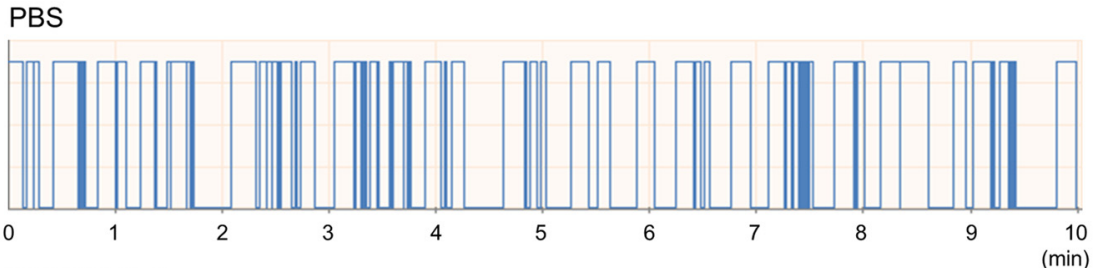

Phrixotoxin-2

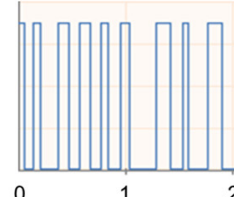

B

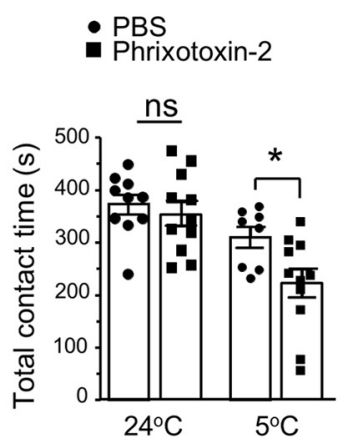

C

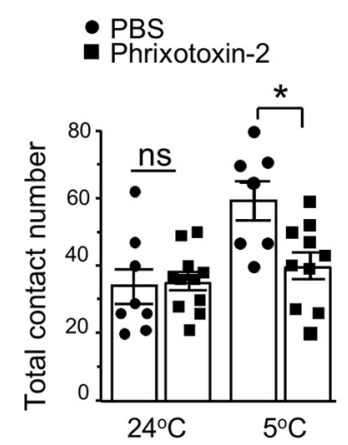

D

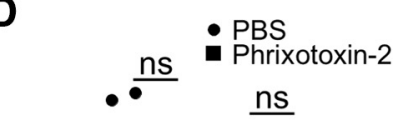

Figure 9. Phrixotoxin-2 induces orofacial cold hypersensitivity. $\boldsymbol{A}$, Sample traces show orofacial operant tests in a rat injected with vehicle (PBS, top) and a different rat following the injection of phrixotoxin-2 (2 $\mu \mathrm{m}, 50 \mu \mathrm{l}$, bottom). Phrixotoxin-2 $(2 \mu \mathrm{m}, 50 \mu \mathrm{l})$ was intracutaneously injected in orofacial regions. The orofacial operant test was performed $60 \mathrm{~min}$ following the injections of PBS or phrixotoxin- 2 and the thermal module was set at $24^{\circ} \mathrm{C}$ or $5^{\circ} \mathrm{C}$. B-D, Summary data of total contact time $(\boldsymbol{B})$, total contact numbers $(\boldsymbol{C})$, and mean contact time $(\boldsymbol{D})$ of the vehicle-injected group $(n=8$, closed circles) and phrixotoxin-2-injected group ( $n=11$, closed squares). Data represent mean \pm SEM; NS, no significant difference; $* p<0.05$.

with control group injected with PBS $(n=8)$. In contrast, there were no significant differences in total contact time and total contact numbers between the control group $(n=8)$ and the phrixotoxin-2-injected group $(n=11)$ when thermal module was set at $24^{\circ} \mathrm{C}$ (Fig. 9B,C). No significant differences in mean contact time were observed between the control group $(n=8)$ and phrixotoxin-2-injected group tested with thermal module set at either $24^{\circ} \mathrm{C}$ or $5^{\circ} \mathrm{C}$ (Fig. 9D).

\section{NS5806 increases IA currents in small-sized V2 TG neurons and alleviates orofacial behavioral cold hypersensitivity in ION-CCI rats}

Since we found that orofacial behavioral cold hypersensitivity in the ION-CCI rats was associated with the reduction of IA currents and downregulation of Kv4.3 channel expression in smallsized V2 TG neurons, we determined whether pharmacological enhancement of Kv4.3 activity could alleviate the orofacial cold hypersensitivity. We first determined whether NS5806, an activator of Kv4.3 channels (Lundby et al., 2010), could potentiate IA currents in small-sized V2 TG neurons of the ION-CCI group. In this set of experiments, patch-clamp recordings were applied to the small-sized V2 TG neurons of the ION-CCI group. Isolated IA currents were recorded in the absence and presence of $50 \mu \mathrm{M}$ NS5806. Of 11 small-sized V2 TG neurons tested, IA currents were shown to be potentiated in seven cells following the application of NS5806 (Fig. 10A-C). Comparison made by using the areas under the I-V curves showed significant increases of IA currents in these small-sized V2 TG neurons by NS5806
(Fig. 10D, $n=7$ ). The remaining four small-sized V2 TG neurons did not show the potentiation by NS5806, most likely because of the lack of Kv4.3 channel expression on these TG neurons.

We next determined whether NS5806 could alleviate orofacial cold hypersensitivity in ION-CCI animals. In this set of experiments, rats of the sham surgery group and ION-CCI group underwent orofacial operant tests following the intraperitoneal administrations of vehicle or $1.67 \mathrm{mg} / \mathrm{kg}$ NS5806. The orofacial operant tests were performed with the thermal module set at $5^{\circ} \mathrm{C}$. Under these conditions, ION-CCI group showed significant reduction of total contact time in comparison with the sham group when vehicle was administered (Fig. 11A,B,D). The total contact time was $146.8 \pm 16.8 \mathrm{~s}(n=6)$ in the sham group, and was significantly reduced to $65.8 \pm 6.2 \mathrm{~s}(n=6, p<0.001)$ in the ION-CCI group (Fig. 11A,B,D). However, following the administration of NS5806 the ION-CCI group had the total contact time of $114.5 \pm 10.8 \mathrm{~s}(n=5)$, significantly longer than that of ION-CCI group injected with vehicle (Fig. 11C,D). The total contact numbers under the above three conditions were not significantly different (Fig. 11E). The mean contact time showed a significant reduction following ION-CCI in comparison with the sham group when vehicle was administered, and the mean contact time in the ION-CCI group was significantly prolonged following the administration of NS5806 (Fig. 11F).

\section{Discussion}

In the present study, we have demonstrated that chronic constrictive injury of infraorbital nerves, the V2 branches of trigeminal nerves, enhances the excitability of the small-sized V2 TG neurons that innervate orofacial cutaneous regions and induces orofacial cutaneous cold hypersensitivity. This cellular and behavioral sensitization is associated with the reduction of IA currents and downregulation of Kv4.3 channels in the smallsized V2 TG neurons in the ION-CCI animals. Furthermore, we have shown that the Kv4.3 blocker phrixotoxin-2 inhibits IA currents in small-sized V2 TG neurons and induces orofacial cold hypersensitivity. On the other hand, the Kv4.3 activator NS5806 (Lundby et al., 2010) enhances IA currents in small-sized V2 TG neurons and alleviates orofacial cold hypersensitivity. Our findings elucidate that Kv4.3 downregulation is an underlying mechanism of the trigeminal neuropathic pain manifested with orofacial cold hypersensitivity. This raises a possibility that targeting Kv4.3 channels with their activators may be clinically useful in treating trigeminal neuropathic pain.

In the present study, some of the electrophysiological recordings were performed in the whole-amount ex vivo TG preparations, which is modified from previous electrophysiological studies using in vitro trigeminal slice preparations (Puil et al., 
1986; Puil and Spigelman, 1988). Our ex vivo TG preparations have allowed us to make wholecell patch-clamp recordings from retrogradelabeled orofacial cutaneous TG afferents and to classify afferent types based on their conduction velocity. However, in our recordings of IA currents, we used acutely dissociated TG neurons to better isolate IA currents. We used phrixotoxin-2 to inhibit and NS5806 to potentiate Kv4.3 channels. However, phrixotoxin-2 also can inhibit and NS5806 can potentiate $\mathrm{Kv} 4.2$ channels (Diochot et al., 1999; Lundby et al., 2010). Nevertheless, Kv4.2 channels have been shown to be minimally expressed in somatosensory neurons (Shinoda et al., 2019). Therefore, these two compounds should primarily target Kv4.3 channels in TG neurons in the present study. Our electrophysiological recordings of IA currents in the present study have limited to the measurements of IA current amplitudes, which allows us to characterize the essential pharmacological properties of these currents and their changes following ION-CCI. More comprehensive characterization of the properties of IA current activation and inactivation (Amberg et al., 2002; Hu et al., 2006; Bourdeau et al., 2007) was not conducted in the present study. In the present study, we conducted our electrophysiological recordings at the room temperature of $24^{\circ} \mathrm{C}$ rather than the body temperature near $37^{\circ} \mathrm{C}$ because TG neurons in ex vivo preparations could stay healthy for longer time at $24^{\circ} \mathrm{C}$ than at $37^{\circ} \mathrm{C}$.

We have previously demonstrated that ION-CCI model is a reliable rat model of trigeminal neuropathic pain and the model well resembles the pain condition following chronic compressions of trigeminal nerves seen clinically (Cha et al., 2012; Abd-Elsayed et al., 2015; Ling et al., 2017). Consistently, the present study also shows the robust behavioral cold hypersensitivity in orofacial areas of ION-CCI rats tested. In the present study, we set the thermal module at $5^{\circ} \mathrm{C}$. With this setting, the skin temperatures in orofacial regions of the testing animals would gradually drop from innocuous to noxious cold, depending on contact time. Therefore, both cold allodynia and hyperalgesia may account for the orofacial behavioral outcomes in our ION-CCI animals. The cold hypersensitivity shown by our orofacial operant behavioral tests in ION-CCI recapitulates the important symptom of trigeminal neuropathic pain seen in human patients, making our study clinically relevant.

The small-sized DiI-labeled V2 TG neurons recorded in the present study are most likely arisen from orofacial cutaneous nociceptive C-afferent fibers based on their conduction velocity and AP shapes. We have shown that these small-sized V2 TG neurons become more excitable following ION-CCI. This is evidenced by the less negative resting membrane potentials, the reduced AP rheobase, and the increased AP firing numbers in these V2 TG neurons following ION-CCI. The enhanced excitability of these small-sized cutaneous V2 TG neurons is consistent with the behavioral cold hypersensitivity in orofacial cutaneous regions shown in the ION-CCI animals. We show that the enhanced excitability of these small-sized V2 TG neurons is associated with the significant reduction of IA currents. Since IA currents in primary afferent neurons counteract membrane depolarization to control neuronal excitability, it is conceivable that a reduction of IA currents following ION-CCI would contribute to the enhanced excitability of the V2 TG neurons. In DRG neurons, IA currents were found to be mediated mainly by $\mathrm{Kv} 1.4, \mathrm{Kv} 3.4, \mathrm{Kv} 4.1$, and $\mathrm{Kv} 4.3$. Of these $\mathrm{Kv}$ channels mediating IA currents, Kv4.3 channels were shown to be mainly expressed on smallsized nociceptive DRG neurons that also expressed TRPV1 receptors and NaV1.8 channels (Huang et al., 2005; Phuket and Covarrubias, 2009). Consistently, we show that Kv4.3 channels were mainly expressed in small-sized V2 TG neurons with nociceptor properties. Thus, Kv4.3-expressing V2 TG neurons are also most likely nociceptive afferent neurons. In the present study, the reduction of IA currents in small-sized V2 TG neurons is consistent with the downregulation of $\mathrm{Kv} 4.3$ channel expression in the V2 TG neurons following ION-CCI. Downregulation of Kv4.3 channel expression has been observed in nociceptive DRG neurons in a neuropathic pain model induced by spinal nerve ligation in rats and in a rat model of hand-arm vibration syndrome (Chien et al., 2007; Conner et al., 2016). The decreases of Kv4.3 expression in nociceptive DRG neurons have been suggested to be an underlying mechanism leading to hyperexcitability of nociceptive DRG neurons and pathologic pain (Chien et al., 2007; Conner et al., 2016). We have recently shown that Kv4.3 channels are downregulated in V2 TG neurons in a chemotherapyinduced neuropathic pain model which manifested with mechanical allodynia and cold allodynia/hyperalgesia 
A Sham/Vehicle

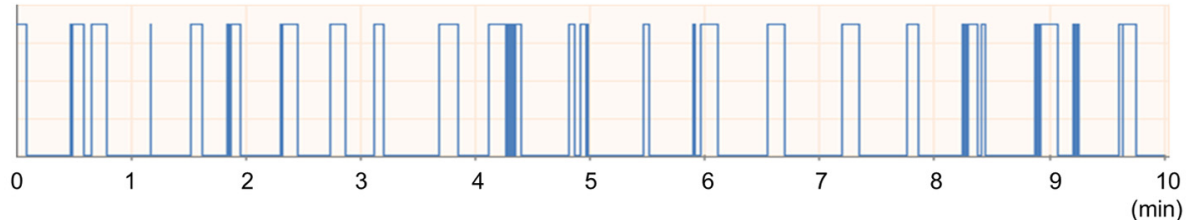

B $\mathrm{ION}-\mathrm{CCl} /$ Vehicle

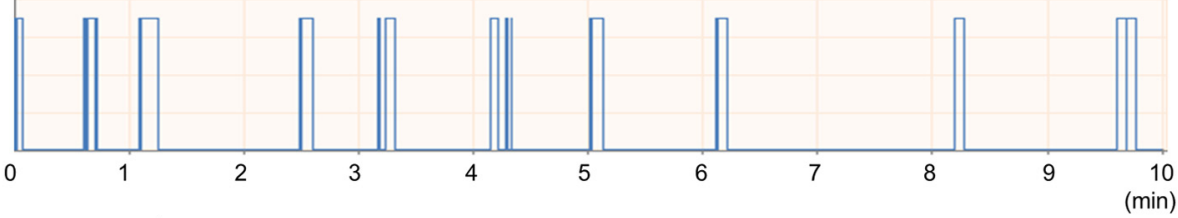

C ION-CCI/NS5806
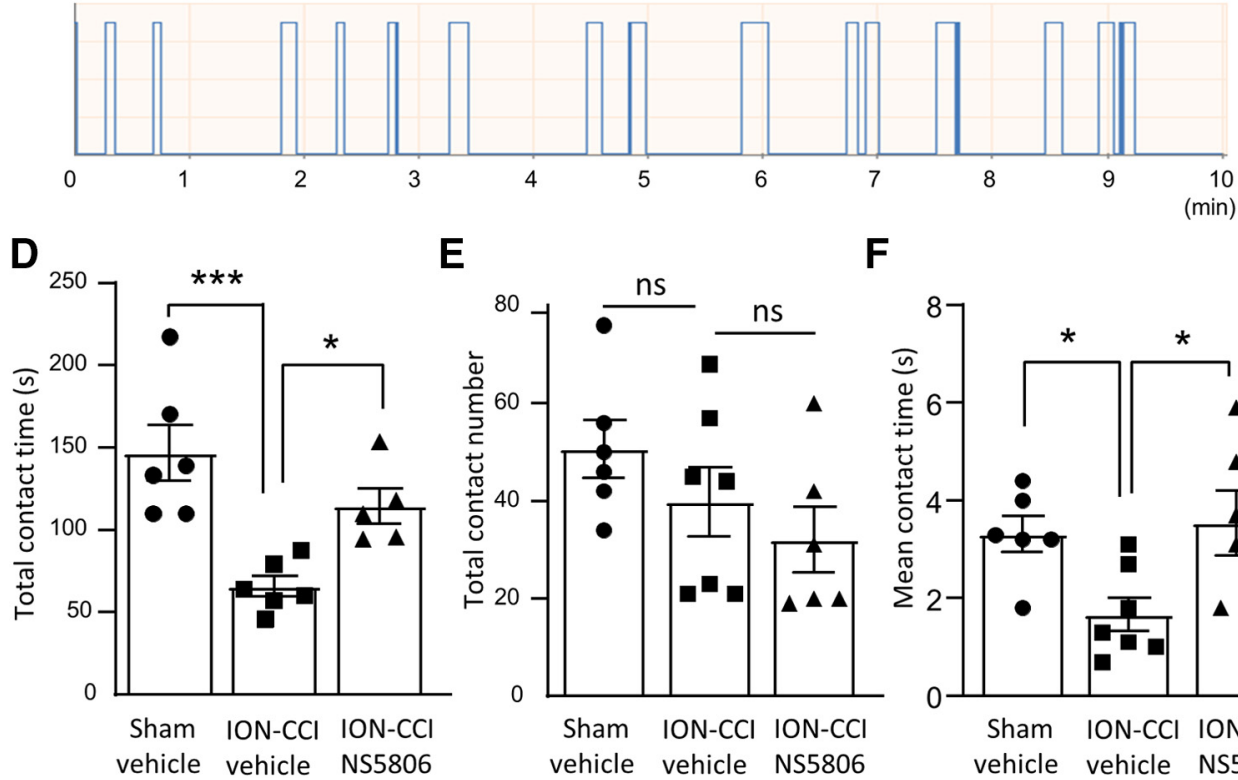

$\mathbf{F}$

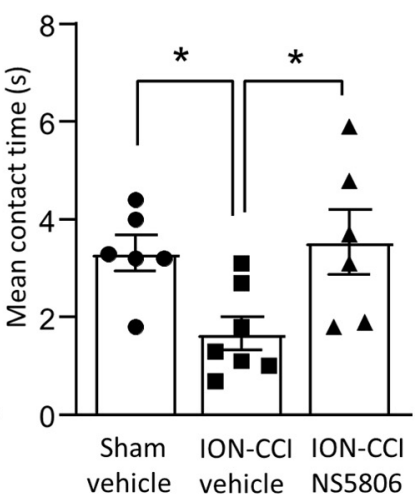

Figure 11. NS5806 alleviates orofacial cold hypersensitivity in ION-CCI rats. $\boldsymbol{A}-\boldsymbol{C}$, Sample traces show orofacial operant tests in a sham rat injected with vehicle $(\boldsymbol{A})$, an ION-C(I rat injected with vehicle $(\boldsymbol{B})$, and an ION-C(I rat injected with NS5806 (C). The thermal module was set at $5^{\circ} \mathrm{C}$ and each animal underwent a 10 min session of the orofacial operant test. $\boldsymbol{D}-\boldsymbol{F}$, Summary data of total contact time $(\boldsymbol{D})$, total contact numbers $(\boldsymbol{E})$, and mean contact time $(\boldsymbol{F})$ of the following three groups of animals, sham group injected with vehicle $(n=6$, closed circles), ION-C(I group injected with vehicle ( $n=6$, closed squares), and ION-CCI group injected with NS5806 ( $n=6$, closed triangles). NS5806 was intraperitoneally injected at $1.67 \mathrm{mg} / \mathrm{kg}$. Data represent mean \pm SEM; NS, no significant difference; $* p<0.05, * * * p<0.001$.

(Viatchenko-Karpinski et al., 2018). In the present study with ION-CCI model, downregulation of Kv4.3 channel expression in V2 TG neurons may contribute to the hyperexcitability of nociceptive V2 TG neurons including the reduction of $\mathrm{AP}$ rheobase, the depolarized resting membrane potentials, and the increased nociceptive V2 TG neurons with the multiple AP firing pattern. This enhanced neuronal excitability may facilitate the occurrence of trigeminal neuropathic pain manifested with cold hypersensitivity.

The present study has focused on Kv4.3 channels because they are mainly expressed in the small-sized nociceptive-like V2 TG neurons. However, it should be noted that ION-CCI may also lead to the downregulation of other voltage-gated $\mathrm{K}^{+}$channels that are involved in nociceptor excitability. In fact, previous studies have shown that downregulation of Kv1 type of voltagegated $\mathrm{K}^{+}$channels underlies cold allodynia following peripheral nerve injury (González et al., 2017). In nociceptive DRG neurons, previous studies have shown that Kv3.4 and Kv1.4 channels are expressed on nociceptive DRG neurons (Rasband et al., 2001;
Huang et al., 2005) and undergo downregulation under pathologic conditions (Alvarez et al., 2019). Therefore, it will be interesting to determine in the future whether these two IA types of $\mathrm{Kv}$ channels may be also expressed on nociceptive-like V2 TG neurons and undergo downregulation following ION-CCI. Kv3.4 channels are activated at high voltages and their primary role is to drive rapid AP repolarization (Zemel et al., 2018). Reduced expression or function of Kv3.4 channels would retard AP repolarization to broaden AP width (Liu et al., 2017). In our study, AP widths of small-sized cutaneous V2 TG neurons became significantly broadened, raising a possibility that this may be because of a downregulation of Kv3.4 following IONCCI.

We show that Kv4.3 channel blocker phrixotoxin-2 decreases IA currents in small-sized V2 TG neurons and induces orofacial cold hypersensitivity. On the other hand, the Kv4.3 channel activator NS5806 (Lundby et al., 2010) enhanced IA currents in the small-sized V2 TG neurons of ION-CCI rats and alleviated cold hypersensitivity in the ION-CCI group. These results strengthen the idea that Kv4.3 downregulation contributes to orofacial cold 
hypersensitivity and restoring Kv4.3 channel activity can prevent the peripheral sensitization. These results also suggest that Kv4.3 channels may serve as therapeutic targets for treating trigeminal neuropathic pain clinically. Since Kv4.3 channel expression is limited to nociceptive type of somatosensory neurons, these channels may provide a desirable pharmacological target for intervening peripheral nociceptive hypersensitization and neuropathic pain. Potent and selective activators of Kv4.3 should be of great interests for future pharmaceutical development of novel pain medicine in treating patients with trigeminal neuropathic pain.

\section{References}

Abd-Elsayed AA, Ikeda R, Jia Z, Ling J, Zuo X, Li M, Gu JG (2015) KCNQ channels in nociceptive cold-sensing trigeminal ganglion neurons as therapeutic targets for treating orofacial cold hyperalgesia. Mol Pain 11:45.

Alvarez P, Bogen O, Levine JD (2019) Interleukin 6 decreases nociceptor expression of the potassium channel KV1.4 in a rat model of hand-arm vibration syndrome. Pain 160:1876-1882.

Amberg GC, Baker SA, Koh SD, Hatton WJ, Murray KJ, Horowitz B, Sanders KM (2002) Characterization of the A-type potassium current in murine gastric antrum. J Physiol 544:417-428.

Bautista DM, Siemens J, Glazer JM, Tsuruda PR, Basbaum AI, Stucky CL, Jordt SE, Julius D (2007) The menthol receptor TRPM8 is the principal detector of environmental cold. Nature 448:204208.

Bendtsen L, Zakrzewska JM, Abbott J, Braschinsky M, Di Stefano G, Donnet A, Eide PK, Leal PRL, Maarbjerg S, May A, Nurmikko T, Obermann M, Jensen TS, Cruccu G (2019) EAN guideline on trigeminal neuralgia. Eur J Neurol 26:831-849.

Bourdeau ML, Morin F, Laurent CE, Azzi M, Lacaille JC (2007) Kv4.3-mediated A-type $\mathrm{K}+$ currents underlie rhythmic activity in hippocampal interneurons. J Neurosci 27:1942-1953.

Castellanos A, Pujol-Coma A, Andres-Bilbe A, Negm A, Callejo G, Soto D, Noël J, Comes N, Gasull X (2020) TRESK background K(+) channel deletion selectively uncovers enhanced mechanical and cold sensitivity. J Physiol 598:1017-1038.

Cha M, Kohan KJ, Zuo X, Ling JX, Gu JG (2012) Assessment of chronic trigeminal neuropathic pain by the orofacial operant test in rats. Behav Brain Res 234:82-90.

Chien LY, Cheng JK, Chu D, Cheng CF, Tsaur ML (2007) Reduced expression of A-type potassium channels in primary sensory neurons induces mechanical hypersensitivity. J Neurosci 27:98559865.

Christoforou J (2018) Neuropathic orofacial pain. Dent Clin North Am 62:565584 .

Colburn RW, Lubin ML, Stone DJ Jr, Wang Y, Lawrence D, D'Andrea MR, Brandt MR, Liu Y, Flores CM, Qin N (2007) Attenuated cold sensitivity in TRPM8 null mice. Neuron 54:379-386.

Conner LB, Alvarez P, Bogen O, Levine JD (2016) Role of Kv4.3 in vibrationinduced muscle pain in the rat. J Pain 17:444-450.

Devine M, Hirani M, Durham J, Nixdorf DR, Renton T (2018) Identifying criteria for diagnosis of post-traumatic pain and altered sensation of the maxillary and mandibular branches of the trigeminal nerve: a systematic review. Oral Surg Oral Med Oral Pathol Oral Radiol 125:526-540.

Diochot S, Drici MD, Moinier D, Fink M, Lazdunski M (1999) Effects of phrixotoxins on the Kv4 family of potassium channels and implications for the role of Itol in cardiac electrogenesis. Br J Pharmacol 126:251-263.

Feller L, Khammissa RAG, Fourie J, Bouckaert M, Lemmer J (2017) Postherpetic neuralgia and trigeminal neuralgia. Pain Res Treat 2017:1681765.

Gada K, Plant LD (2019) Two-pore domain potassium channels: emerging targets for novel analgesic drugs: IUPHAR Review 26. Br J Pharmacol 176:256-266.

González A, Ugarte G, Restrepo C, Herrera G, Piña R, Gómez-Sánchez JA, Pertusa M, Orio P, Madrid R (2017) Role of the excitability brake potassium current IKD in cold allodynia induced by chronic peripheral nerve injury. J Neurosci 37:3109-3126.

$\mathrm{Hu}$ HJ, Carrasquillo Y, Karim F, Jung WE, Nerbonne JM, Schwarz TL, Gereau RWt (2006) The kv4.2 potassium channel subunit is required for pain plasticity. Neuron 50:89-100.

Huang HY, Cheng JK, Shih YH, Chen PH, Wang CL, Tsaur ML (2005) Expression of A-type K channel alpha subunits Kv 4.2 and $\mathrm{Kv} 4.3$ in rat spinal lamina II excitatory interneurons and colocalization with pain-modulating molecules. Eur J Neurosci 22:11491157.

Knowlton WM, Palkar R, Lippoldt EK, McCoy DD, Baluch F, Chen J, McKemy DD (2013) A sensory-labeled line for cold: TRPM8expressing sensory neurons define the cellular basis for cold, cold pain, and cooling-mediated analgesia. J Neurosci 33:28372848.

Ling J, Erol F, Viatchenko-Karpinski V, Kanda H, Gu JG (2017) Orofacial neuropathic pain induced by oxaliplatin: downregulation of KCNQ2 channels in V2 trigeminal ganglion neurons and treatment by the KCNQ2 channel potentiator retigabine. Mol Pain 13:1744806917724715.

Liu PW, Blair NT, Bean BP (2017) Action potential broadening in capsaicinsensitive DRG neurons from frequency-dependent reduction of Kv3 current. J Neurosci 37:9705-9714.

Lundby A, Jespersen T, Schmitt N, Grunnet M, Olesen SP, Cordeiro JM, Calloe K (2010) Effect of the I(to) activator NS5806 on cloned $\mathrm{K}(\mathrm{V}) 4$ channels depends on the accessory protein KChIP2. Br J Pharmacol 160:2028-2044.

Madrid R, de la Peña E, Donovan-Rodriguez T, Belmonte C, Viana F (2009) Variable threshold of trigeminal cold-thermosensitive neurons is determined by a balance between TRPM8 and Kv1 potassium channels. J Neurosci 29:3120-3131.

McKemy DD (2005) How cold is it? TRPM8 and TRPA1 in the molecular logic of cold sensation. Mol Pain 1:16.

McKemy DD, Neuhausser WM, Julius D (2002) Identification of a cold receptor reveals a general role for TRP channels in thermosensation. Nature 416:52-58.

Morenilla-Palao C, Luis E, Fernández-Peña C, Quintero E, Weaver JL, Bayliss DA, Viana F (2014) Ion channel profile of TRPM8 cold receptors reveals a role of TASK-3 potassium channels in thermosensation. Cell Rep 8:1571-1582.

Peier AM, Moqrich A, Hergarden AC, Reeve AJ, Andersson DA, Story GM, Earley TJ, Dragoni I, McIntyre P, Bevan S, Patapoutian A (2002) A TRP channel that senses cold stimuli and menthol. Cell 108:705-715.

Phuket TR, Covarrubias M (2009) Kv4 channels underlie the subthresholdoperating A-type K-current in nociceptive dorsal root ganglion neurons. Front Mol Neurosci 2:3

Piña R, Ugarte $G$, Campos $M$, Íñigo-Portugués $A$, Olivares $E$, Orio $P$, Belmonte C, Bacigalupo J, Madrid R (2019) Role of TRPM8 channels in altered cold sensitivity of corneal primary sensory neurons induced by axonal damage. J Neurosci 39:8177-8192.

Puil E, Spigelman I (1988) Electrophysiological responses of trigeminal root ganglion neurons in vitro. Neuroscience 24:635-646.

Puil E, Gimbarzevsky B, Miura RM (1986) Quantification of membrane properties of trigeminal root ganglion neurons in guinea pigs. J Neurophysiol 55:995-1016.

Qu L, Caterina MJ (2016) Enhanced excitability and suppression of A-type K $(+)$ currents in joint sensory neurons in a murine model of antigeninduced arthritis. Sci Rep 6:28899.

Rasband MN, Park EW, Vanderah TW, Lai J, Porreca F, Trimmer JS (2001) Distinct potassium channels on pain-sensing neurons. Proc Natl Acad Sci USA 98:13373-13378.

Sarria I, Ling J, Gu JG (2012) Thermal sensitivity of voltage-gated Na+ channels and A-type $\mathrm{K}+$ channels contributes to somatosensory neuron excitability at cooling temperatures. J Neurochem 122:1145-1154.

Sharif-Naeini R (2020) TRESK is a modality-specific brake on nociceptor excitability. J Physiol 598:1423-1424.

Shinoda M, Fukuoka T, Takeda M, Iwata K, Noguchi K (2019) Spinal glial cell line-derived neurotrophic factor infusion reverses reduction of Kv4.1-mediated A-type potassium currents of injured myelinated 
primary afferent neurons in a neuropathic pain model. Mol Pain 15:174480691984119.

Stucky CL, Dubin AE, Jeske NA, Malin SA, McKemy DD, Story GM (2009) Roles of transient receptor potential channels in pain. Brain Res Rev 60:2-23.

Takashima Y, Daniels RL, Knowlton W, Teng J, Liman ER, McKemy DD (2007) Diversity in the neural circuitry of cold sensing revealed by genetic axonal labeling of transient receptor potential melastatin 8 neurons. J Neurosci 27:14147-14157.

Tan ZY, Donnelly DF, LaMotte RH (2006) Effects of a chronic compression of the dorsal root ganglion on voltage-gated $\mathrm{Na}+$ and $\mathrm{K}+$ currents in $\mathrm{cu}-$ taneous afferent neurons. J Neurophysiol 95:1115-1123.

Viatchenko-Karpinski V, Ling J, Gu JG (2018) Down-regulation of Kv4.3 channels and a-type $\mathrm{K}(+)$ currents in V2 trigeminal ganglion neurons of rats following oxaliplatin treatment. Mol Pain 14:1744806917750995.
Waxman SG, Zamponi GW (2014) Regulating excitability of peripheral afferents: emerging ion channel targets. Nat Neurosci 17:153-163.

Xing H, Chen M, Ling J, Tan W, Gu JG (2007) TRPM8 mechanism of cold allodynia after chronic nerve injury. J Neurosci 27:1368013690.

Zakrzewska JM (2013) Differential diagnosis of facial pain and guidelines for management. Br J Anaesth 111:95-104.

Zakrzewska JM, McMillan R (2011) Trigeminal neuralgia: the diagnosis and management of this excruciating and poorly understood facial pain. Postgrad Med J 87:410-416.

Zemel BM, Ritter DM, Covarrubias M, Muqeem T (2018) A-type KV channels in dorsal root ganglion neurons: diversity, function, and dysfunction. Front Mol Neurosci 11:253.

Zuo X, Ling JX, Xu GY, Gu JG (2013) Operant behavioral responses to orofacial cold stimuli in rats with chronic constrictive trigeminal nerve injury: effects of menthol and capsazepine. Mol Pain 9:28. 